\title{
Reliability-based design of a slat track fatigue life using mesh morphing technology
}

\author{
Roberto d'Ippolito* and Stijn Donders ${ }^{\dagger}$ \\ LMS International, Interleuvenlaan 68, B-3001 Leuven, Belgium \\ Michael Hack ${ }^{*}$ \\ LMS Deutschland GmbH, Kaiserslautern, Germany \\ Nick Tzannetakis ${ }^{\S}$ \\ NOESIS Solutions, Interleuvenlaan 68, B-3001 Leuven, Belgium \\ Geert Van der Linden ** \\ ASCO Industries, Weiveldlaan 2, B1930 Zaventem, Belgium \\ and \\ Dirk Vandepitte ${ }^{\dagger \dagger}$ \\ Katholieke Universiteit Leuven, Department of Mechanical Engineering, \\ Division PMA, B-3001 Heverlee, Belgium
}

\begin{abstract}
Although the aerospace production process is much better controlled than in other industries, it remains true that very small manufacturing tolerances exist in the geometrical parameters (flange thicknesses, hole diameters, ...). In the current design process, the effect of this manufacturing variability on the structural durability and safety cannot be accurately assessed and is hence compensated for by applying safety factors. This is not an ideal situation, as it may lead to slightly over-designed structures. A much more promising approach is to include probabilistic models of design variables into the mechanical simulation process. Then, with a new methodology based on reliability analysis, engineers can obtain a better understanding of the actual effect of the manufacturing tolerances and of variability in material properties. Based on the analysis results, the robustness and reliability of the design can be assessed and improved if needed. In this paper, the abovementioned probabilistic approach is demonstrated on a slat track structure. Measurements of different geometrical properties have been collected during the manufacturing process and their variability has been characterized probabilistically with statistical models. Then, a reliability analysis has been carried out using mesh morphing technology and fatigue life predictions with an industrial-sized FE model of the slat track to assess the reliability of the structure in terms of fatigue life. The outcome of the analysis consists of a probabilistic model of the structural performance (e.g. fatigue life for the slat track), given the variability in the geometrical parameters. Then, a reliability-based design optimization procedure has been carried out to improve the design of the slat track, while maintaining the same reliability of the nominal design.
\end{abstract}

\footnotetext{
* Research Engineer, CAE Division, Interleuvenlaan 68, AIAA Student Member.

${ }^{\dagger}$ Research Engineer, CAE Division, Interleuvenlaan 68.

* Product Manager Durability CAE, Kaiserslautern, Germany

${ }^{\S}$ CTO, Interleuvenlaan 68, Leuven, B-3000 Belgium.

${ }^{* *}$ Research \& Development Engineer, Weiveldlaan 2, B1930 Zaventem, Belgium

${ }^{\dagger \dagger}$ Professor, Department of Mechanical Engineering, Division PMA, Kasteelpark Arenberg 41, AIAA Senior Member.
} 


\section{Introduction}

CURRENTLY, aerospace industries are dedicating much attention to improve product quality and reliability through a virtual simulation environment. Although the aerospace production process is much better controlled than the process in other industries, it remains true that very small manufacturing tolerances exist in the geometrical parameters (flange thicknesses, hole diameters, ...) as well as small variabilities in physical properties. In the current design process, the effect of this variability on the static performance or on the structural durability and safety cannot be accurately assessed and is hence compensated for by applying safety factors. This is not an ideal situation, as it may lead to slightly over-designed structures. In order to integrate the simulation environment and the production variability, a much more promising approach has been worked out that includes probabilistic models of design variables into the mechanical simulation process. In this way, the new probabilistic information, gathered from the production process and introduced in the simulation environment, allows the design engineer to obtain a better understanding of the actual effect of the manufacturing tolerances on structural safety. In this context, variability quantification [1][2][3] has been carried out in order to enable the application of the probabilistic techniques available for reliability based design [4][5]. These techniques, developed in the last 30 years for a number of different cases, allow the characterization of variability in structural parameters (geometry, material properties, etc.) and the analysis of its propagation on the final response of the structure [6]. This effect can be studied using robust design, reliability analysis and reliability-based design optimization strategies that make use of limit state approximations as well as of sampling procedures, thus giving to the analyst the possibility to balance the accuracy and the computational effort required to extract the necessary information on safety and performance stability.

In this paper, a fatigue analysis of a slat track using a validated Finite Element (FE) model is considered, being representative of a field where a better understanding of the effect of variability is required. For this model, a probabilistic characterization of the variability has been introduced in the Computer Aided Engineering (CAE) process using advanced mesh morphing technologies. Based on this improved simulation environment, a statistical characterization of the fatigue performance of the structure has been assessed. This characterization allows the use of reliability analysis techniques to predict the probability of violating a selected performance limit. Moreover, a reliability-based design optimization procedure has been carried out to improve the design of the slat track by minimizing the total mass, while maintaining the same reliability level of the nominal design. 


\section{Analysis Case: Slat Track}

The FE core model (Figure 1) consists of 236.630 tetrahedral elements, generated by an automated mesh generator. Beam elements have been added to correctly represent the rack in the inner section of the slat track profile and to model the rod-leading edge assembly, connected by ball bearings.

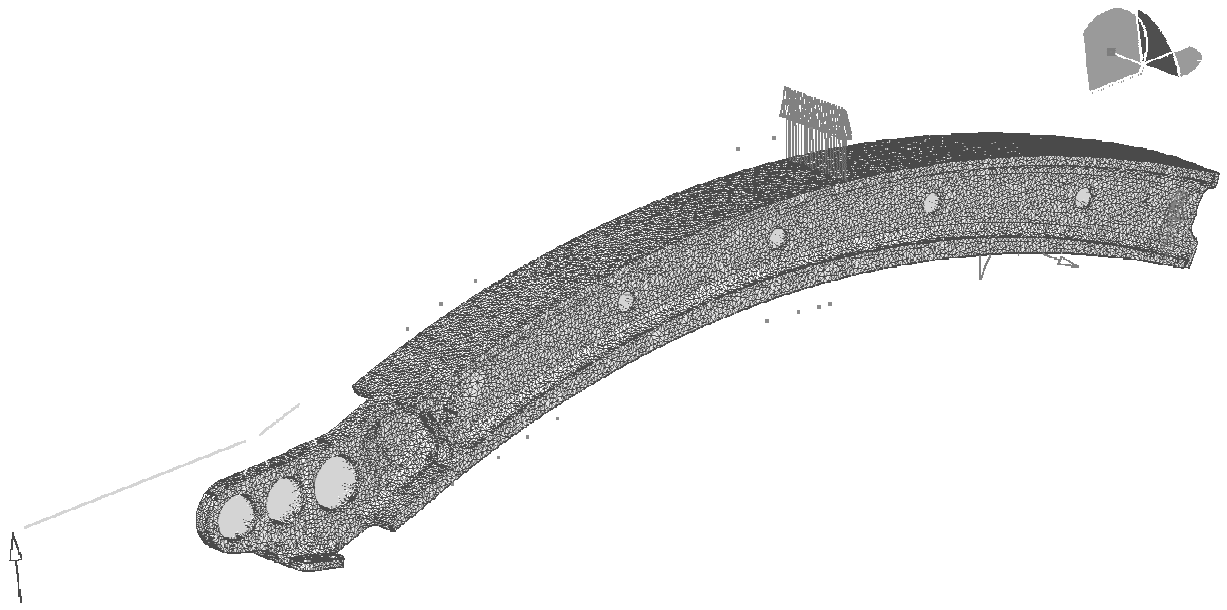

Figure 1: Slat Track FE model with loads and constraints

The slat track is made of a $18 \%$ nickel-cobalt-molybdenum maraging steel, which has an ultimate tensile strength equal to $1800 \mathrm{MPa}$ and a Young's Modulus of $240000 \mathrm{MPa}$.

\section{A. Measured data and probabilistic characterization}

Probabilistic analysis requires that a representative set of data is available to characterize the geometrical variabilities of the manufacturing process. Since every manufacturing process is different from the other, there are no standard assumptions that can be made on the type of distribution to be used to characterize geometric tolerances. This is, of course, not the case for physical quantities, like material properties, that can be easily modeled and where well-known guidelines are available for a wide class of materials.

For the slat track, 4 input parameters were selected for the probabilistic characterization, as demonstrated in Figure 2 . 


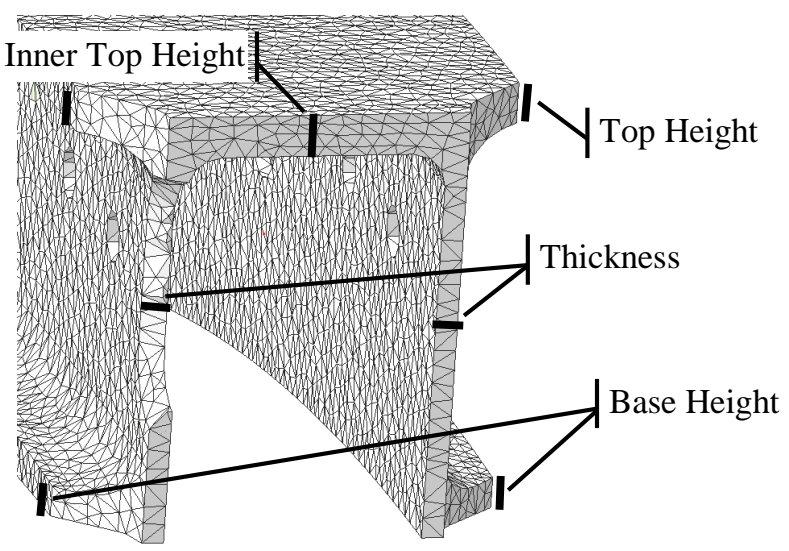

Figure 2: Slat track uncertain parameters

For these parameters, geometrical measurements have been taken on 1573 specimens of nominally identical slat tracks during the manufacturing process and on both sides of the slat track dimensions. In particular, the difference between the nominal (design) value and the actual value has been measured for each parameter. This data has then been used to build a probabilistic characterization of the input parameters using random variable. For this purpose, a least squares fitting procedure has been used with the four available sets of data to find the best statistical distributions that represent the population of measured differences. The type of distribution, its mean and standard deviation are reported in Table 1. The distribution models have been verified with a $\chi^{2}$ hypothesis test [7], which checks that the selected model is representative of the data set used for the least squares procedure.

\begin{tabular}{|c|c|c|c|c|}
\hline Variable & Design Value & Distribution & $\begin{array}{l}\text { Mean of difference } \\
\text { from nominal }\end{array}$ & $\begin{array}{l}\text { Standard Deviation of } \\
\text { difference from nominal }\end{array}$ \\
\hline Base Height & Undisclosed & Normal & $0.15181 \mathrm{~mm}$ & $0.05918 \mathrm{~mm}$ \\
\hline Thickness & Undisclosed & Normal & $0.14722 \mathrm{~mm}$ & $0.04942 \mathrm{~mm}$ \\
\hline Top Height & Undisclosed & Normal & $0.02503 \mathrm{~mm}$ & $0.07012 \mathrm{~mm}$ \\
\hline Inner Top Height & Undisclosed & Normal & $0.10272 \mathrm{~mm}$ & $0.06084 \mathrm{~mm}$ \\
\hline
\end{tabular}

Table 1: Statistical characterization of the input parameters, based on measured data

A check on the statistical correlation of the design parameters was also carried out using the data acquired during the manufacturing process. This check needs to be performed in order to avoid that a possible assumption of statistical independency affects the simulation results. Thus, the estimation $\hat{\rho}_{X Y}$ of the linear correlation coefficient 
$\rho_{X Y}$ has been carried out for the input parameters considered in this case. The definition of the approximate linear correlation coefficient $\hat{\rho}_{X Y}$ between two data sets $X$ and $Y$ of $n$ samples each is (in the least squares sense) [8]:

$$
\hat{\rho}_{X Y}=\frac{\hat{\operatorname{Cov}}\{X, Y\}}{\sqrt{\hat{\operatorname{Var}}\{X\} \hat{\operatorname{Var}}\{Y\}}}=\frac{\frac{1}{n} \sum_{i=1}^{n}\left(x_{i} y_{i}\right)-\mu_{X} \mu_{Y}}{\sqrt{\left(\frac{1}{n} \sum_{i=1}^{n} x_{i}^{2}-\mu_{X}^{2}\right)\left(\frac{1}{n} \sum_{i=1}^{n} y_{i}^{2}-\mu_{Y}^{2}\right)}}
$$

Where $\hat{\operatorname{Cov}}\{X, Y\}$ is the approximate covariance estimated using the realizations of the two random variables;

$\hat{\operatorname{Var}}\{X, Y\}$ is the approximate variance estimated using the realizations of the two random variables.

In Table 2 the values of the approximate correlation coefficients for the design parameters of Table 1 are reported (correlation matrix).

\begin{tabular}{|l|c|c|c|c|}
\hline & Base Height & Thickness & Top Height & Inner Top Height \\
\hline Base Height & 1 & 0.0944 & -0.4679 & -0.1123 \\
\hline Thickness & 0.0944 & 1 & -0.1774 & 0.0563 \\
\hline Top Height & -0.4679 & -0.1774 & 1 & 0.1694 \\
\hline Inner Top Height & -0.1123 & 0.0563 & 0.1694 & 1 \\
\hline
\end{tabular}

Table 2: Statistical correlation between the input parameters, based on measured data

As it can be seen from the correlation matrix, most of the parameters have little or no correlation, except between the Base Height and the Top Height parameters, which show a negative correlation. The causes of this correlation were not investigated, as they mainly depend on the manufacturing tool used for the slat track production, but their effect has been taken into account during the reliability computations. More details will be given in the results section. In Figure 3 the correlation plots for all the parameters are reported. The presence of few outlier samples (defined as observations that lie at an abnormal distance from other values in a statistical population of samples) was neglected for the computation of the correlation matrix. 


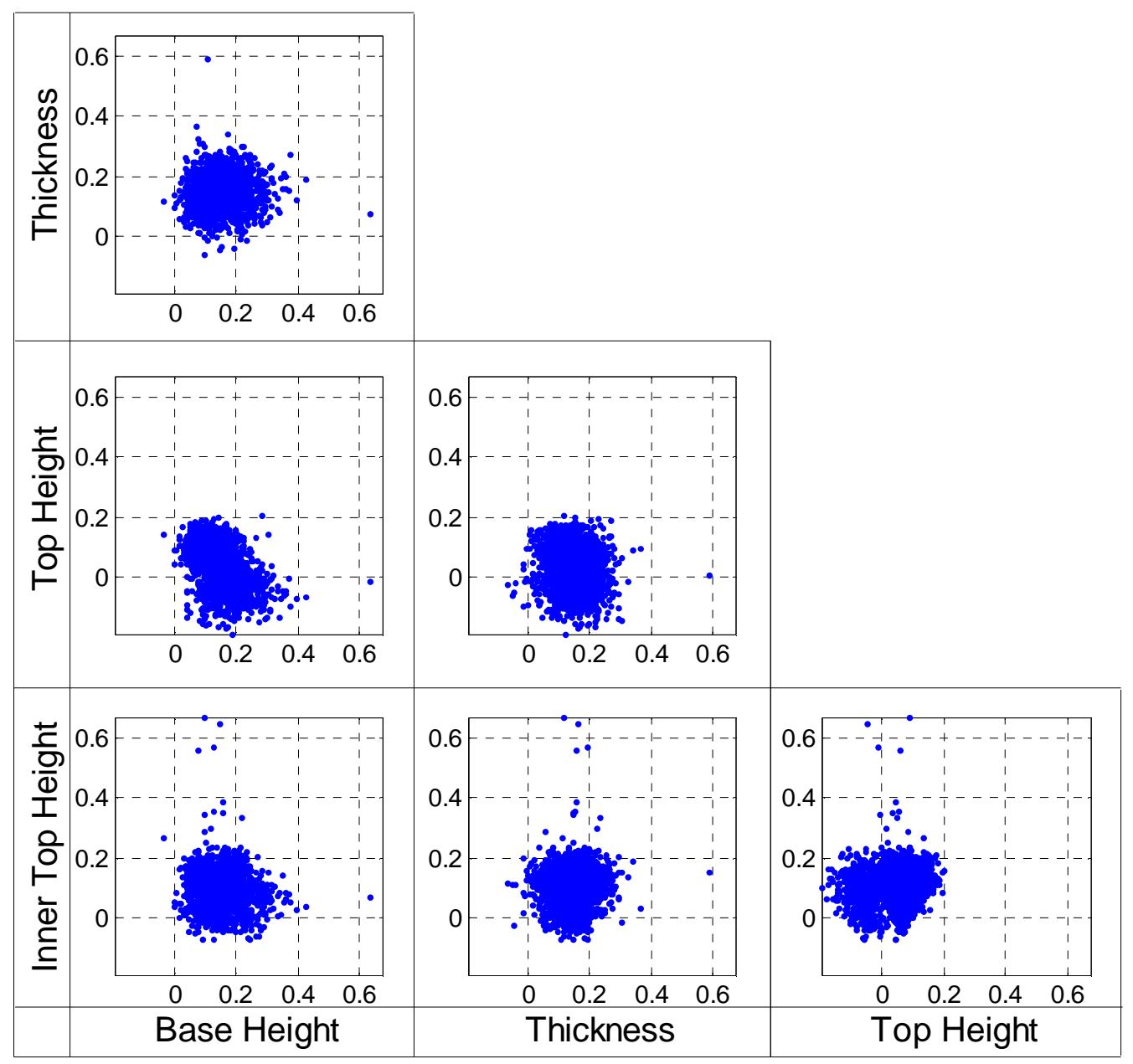

Figure 3: Correlation plots for the design parameters

\section{B. Objective of the analysis}

In this paper, the presence of variability in the input parameters has been taken into account to analyze the fatigue life of the slat track using the FE model. The propagation of variability in the structural analysis and its effect on the component performance has been analyzed and an optimization procedure based on the obtained results has been carried out to improve the weight of the structure, reducing the total mass while keeping the same reliability level. The statistical characterization of the input parameters and their measured correlation has been used in the computation of the fatigue life, thus allowing the application of reliability analysis and reliability-based design optimization techniques using probabilistic constraint formulations. 


\section{The reliability problem statement}

To better understand the methodology used in the analysis, a brief overview of the reliability problem statement is presented here. In the reliability theory, variabilities of an engineering design can be characterized by the variations of a random system parameter set [4]. These random variables are modeled with probability distribution functions and are representative of the uncertain model parameters. Thus, given a set of random variables $x=\left[x_{i}\right]^{T}$, with $\mathrm{i}=1 \ldots n$, the probability distribution of each random variable $x_{i}$ is described either by its cumulative distribution function (CDF, $\left.F_{x_{i}}\left(x_{i}\right)\right)$ or by its probability density function (PDF, $f_{x_{i}}\left(x_{i}\right)$ ) and is often bounded by tolerance limits on the system parameter values [5].

For each realization of the set of input random variables, the performance of the system has to be evaluated. This is generally described by Performance Functions ( $\mathrm{PF}, G_{j}(x)$ with $j=1 \ldots m$ ) that, for a structural design, are usually the selected failure criteria. Thus, if $G_{j}(x)$ is one of the $m$ system PFs, the system is considered to fail if $G_{j}(x)<0$ for at least one index $j$. The probability of failure of the system for every $j^{\text {th }}$ performance function is then the multi dimensional integral of the joint PDF function of the set of random variables over the failure domain $\Omega_{j}$. This is expressed by the integral [4]:

$$
F_{G_{j}}(0)=P\left(G_{j}(x)<0\right)=\int_{\Omega_{j}} \ldots \int f_{X}(x) d x_{1} \ldots d x_{n} \quad \text { where } \Omega_{j}:\left\{x \in \mathfrak{R}^{n}: G_{j}(x)<0\right\}
$$

The event space $\Omega_{j}$ is the region of the stochastic space where only failure events occur. Thus, the integral of the joint probability density function $f_{X}(x)$ over $\Omega_{j}$ yields the probability of failure $P_{f, j}$ of the structure for the $j^{\text {th }}$ failure criterion.

$$
P_{f, j}=F_{G_{j}}(0)=P\left(G_{j}(x)<0\right) \quad \text { with } j=1 \ldots m
$$

In general, given a fixed performance index (or measure) $g_{j}$ of the system, Eq (1) can be generalized by computing the probability of exceedance of the selected $g_{j}$ threshold.

$$
F_{G_{j}}\left(g_{j}\right)=P\left(G_{j}(x)<g_{j}\right)=\int_{\Omega_{G_{j}}} \ldots \int f_{X}(x) d x_{1} \ldots d x_{n} \quad \text { where } \Omega_{G_{j}}:\left\{x \in \Re^{n}: G_{j}(x)<g_{j}\right\}
$$


In this case, the event space $\Omega_{G_{j}}$ represents the region of the stochastic space where the performance parameter of the structure is below the prescribed quantity $g_{j}$.

The main aim of the reliability analysis is to estimate the probability of failure of a structure, given a set of input random variables and a set of failure criteria defining the safe and fail regions $\Omega_{j}$ or $\Omega_{G_{j}}$. This estimation is usually done by approximating the multi dimensional integral with appropriate techniques. These include sampling methods and limit state approximations, usually computed by transforming the problem definition from the parameter space $X$ into the standard normal space $Y$.

The total probability of failure of the structure is given by the integral of the joint PDF $f_{X}(x)$ over the union of all the failure domains. These failure domains are defined by the corresponding Performance Functions $G_{j}(x)$ and their threshold values $g_{j}$ (performance indexes or measures).

In the next sections an overview of the techniques used to approximately estimate the multidimensional integral and used to exploit this estimation for optimization procedures is presented.

\section{A. Limit State Approximations (LSA)}

In most industrial cases, the integrals of Eq. (1) and of Eq. (2) cannot be evaluated in closed form. Only for simple academic cases $f_{X}$ can be defined analytically. In such a case, the performance function $G_{j}(x)$ is available for each specific failure mode of the structure so that $\Omega_{j}$ can be defined. The boundary of the domain $\Omega_{j}$ is also called limit-state surface and the function $G_{j}(x)$ is called the limit-state function (LSF) [5].

In the most widely used reliability methods, approximations are made in the space of standard uncorrelated normal variates, $Y$, obtained from a transformation of the basic variables

$$
Y=T(X)
$$

where the transformation $T$ is expressed in terms of the distributions of $X$.

In the space $Y$, denoted as the standard normal space, approximations of the probability of failure $P_{f, j}$ of Eq. (1) are obtained by replacing the limit state surface with first or second order approximating surfaces. These surfaces are fitted to the limit state surface at points with minimal distance to the origin (design points). 


\section{B. The Reliability-Based Design Optimization (RBDO) Model}

In engineering design, the traditional deterministic design optimization model has been successfully applied to systematically improve the system design process, yielding a reduction of the costs and an improvement of the final quality of the products. However, uncertainties in either engineering simulations and/or manufacturing processes exist. This calls for different optimization models that can yield not only an improvement in the design, but also a higher level of confidence. Thus, a reliability-based design optimization (RBDO) model for robust and costeffective designs can be defined using mean values of the random system variables as design parameters and optimizing the cost subject to prescribed probabilistic constraints (like probabilities of failure) by solving a mathematically nonlinear programming problem.

The general RBDO model can be defined as

$$
\left\{\begin{array}{l}
\min _{d}\left\{\operatorname{Cost}\left[d\left(\mu_{X}\right)\right]\right\} \\
\text { subject to } P_{f, j}=P\left(G_{j}(x)<0\right) \leq \bar{P}_{f, j} \text { with } j=1 \ldots m
\end{array}\right.
$$

where

- the cost function can be any function of the design $d\left(\mu_{X}\right)$

- the design $d\left(\mu_{X}\right)$ depends only on the mean values $\mu_{X}$ of the input parameters and

- $\quad \bar{P}_{f, j}$ is the probabilistic constraint that can be defined for each failure mode $j$ and needs to be satisfied.

For this optimization problem, the constraint definition is expressed in terms of probability distributions and thus needs to be evaluated, for each optimization step, within the probability framework. Thus, for each iteration of the optimization loop, an estimation of the probabilistic constraint in terms of its multidimensional integral (see Eq. (1) and (2)) has to be computed. For this purpose, different methods exist. Most of them apply a transformation of the input parameter space $X$ to the standard normal space $Y$. In this space, if the limit state functions are linear, each probability of failure $P_{f, j}$ can be represented in terms of the reliability index $\beta_{j}[4]$ as

$$
P_{f, j}=P\left(G_{j}(x)<0\right) \Rightarrow P_{f, j}=P\left(G_{j}(y)<0\right)=\Phi\left(-\beta_{j}\right) \Rightarrow \beta_{j}=-\Phi^{-1}\left(P_{f, j}\right)
$$

Where $\Phi(\bullet)$ is the standard normal CDF (zero mean and standard deviation 1).

This equation can be generalized as

$$
F_{G_{j}}\left(g_{j}\right)=P\left(G_{j}(x)<g_{j}\right)=\Phi\left(-\beta_{G_{j}}\right)
$$


Where $F_{G_{j}}(\bullet)$ is the $\mathrm{CDF}$ of the $j^{\text {th }}$ system response.

The same approach can be used also to express the probabilistic constraint of Eq. (4) in a different notation. In this case, the probability of failure $P_{f, j}$ will be the target probability of failure $\bar{P}_{f, j}$ and the reliability index $\beta_{j}$ the target reliability index $\beta_{t, j}$.

$$
\bar{P}_{f, j}=\Phi\left(-\beta_{t, j}\right) \Rightarrow \beta_{t, j}=-\Phi^{-1}\left(\bar{P}_{f, j}\right)
$$

Using Eq. (6), the second condition of Eq. (4) can be rewritten as

$$
P_{f, j}=F_{G_{j}}(0)=\Phi\left(-\beta_{j}\right) \leq \Phi\left(-\beta_{t, j}\right)=\bar{P}_{f, j} \Rightarrow \beta_{j} \geq \beta_{t, j}
$$

The relation expressed in Eq. (8) in terms of the reliability index, can also be expressed in terms of the performance measure through inverse transformation. In fact, using Eq. (7) and Eq. (8), the target probability of failure can be expressed in terms of the target performance measure as

$$
\bar{g}_{j}=F_{G_{j}}^{-1}\left(\bar{P}_{f, j}\right)=F_{G_{j}}^{-1}\left[\Phi\left(-\beta_{t, j}\right)\right]
$$

where $\bar{g}_{j}$ is named "target probabilistic performance measure". It represents the value of the performance function "equivalent" to the target reliability index $\beta_{t, j}$.

The expression of the probabilistic constraint of Eq. (8) and (9) can be used in the optimization problem of Eq. (4) to equivalently replace the original definition.

\section{Performance Measure Approach (PMA)}

The formulation of the general RBDO model of Eq. (3) that uses Eq. (6) to describe the probabilistic constraint is called Reliability Index Approach (RIA) [9][10]. An alternative and more effective way of formulating the general RBDO model is to use the inverse formulation of the probabilistic constraint of Eq. (7), known as Performance Measure Approach (PMA) [11][12]. The RBDO model can be re-written as

$$
\left\{\begin{array}{l}
\min _{d}\left\{\operatorname{Cost}\left[d\left(\mu_{X}\right)\right]\right\} \\
\text { subject to } \bar{g}_{j} \geq g_{j} \text { with } j=1 \ldots m
\end{array}\right.
$$

In this case, at a given design $d^{(k)}=\left[d_{i}^{(k)}\right]^{T} \equiv\left[\mu_{i}^{(k)}\right]^{T}$, the evaluation of the target probabilistic performance measure $\bar{g}_{j}$ is performed using 


$$
\bar{g}_{j}\left(d^{(k)}\right)=F_{G_{j}}^{-1}\left(\int_{\Omega_{G_{j}}} \ldots \int f_{X}(x) d x_{1} \ldots d x_{n}\right)
$$

To facilitate the estimation of the integral in Eq. (11), a transformation from the parameter space $X$ to the standard normal uncorrelated space $Y$ is generally used [12]. This permits to easily evaluate the integral by transforming the joint PDF function of the input random variables in a multidimensional uncorrelated joint normal PDF, which can be evaluated more easily.

Thus, for each iteration of the optimization process the following problem has to be solved

$$
\left\{\begin{array}{l}
\min _{u}\left[G_{j}(y) \mid\right] \text { with } j=1 \ldots m \\
\text { subject to }\|y\|=\beta_{t, j}
\end{array}\right.
$$

Where $G(y)$ is defined by the transformation $Y=T(X)$ from the input parameters space $X$ to the standard normal space $Y$. The approximate solution of this problem yields an estimation of the performance measure, given by

$$
\bar{g}_{j} \approx\left\|G\left(\bar{y}_{\beta=\beta_{t, j}}\right)\right\|
$$

With the formulation used in Eq. (13), the performance measure $\bar{g}_{j}$, which depends on the mean values of the input random parameters (also called nominal point), has to be estimated for each iteration. The target of the optimization is to find the nominal point that can satisfy all the probabilistic constraints in terms of the performance measure. This approach yields an improved convergence rate of the PMA methodology with respect to the RIA formulation. In fact, PMA minimizes a complex cost function subject to a simple constraint, which raises less numerical problems and therefore yields a better algorithm performance, while the RIA algorithm minimizes a simple cost function subject to a complex constraint.

Both these methods use a Limit State Approximation to make an approximation of the exact Limit State Function (LSF); refer to [4][13] for a more elaborate description. In particular, the method considered in this paper is the First Order Reliability Method (FORM) and it has been used for both RIA and PMA. This method has the advantage of being fast and relatively accurate in the analysis for less than 15 input variables [14]. However, it is recognized that such an approach can lead to large approximation errors for a higher number of variables, and also to long computation times when using a gradient-based sensitivity computation [15]. Considering such highdimensional problems is however outside the scope of this paper. 
For the sake of simplicity, only the case with one failure criterion $(j=l)$ will be considered in this paper, therefore the $j$ index is removed from all the quantities that depend on $j$.

\section{First and Second Order Reliability Methods}

In the First Order Reliability Method (FORM), the limit state surface is replaced with its tangent hyperplane in the standard normal space $Y$ at the global minimum distance point (Most Probable Point, MPP) on the limit state surface. With this approximation, the reliability index $\beta_{s}$ coincides with the distance of the MPP to the origin. In the Second Order Reliability Method (SORM) the limit state surface is approximated by a paraboloid, defined with the same principal curvatures as estimated for the limit state surface at the design point (MPP). Determination of these curvatures essentially requires computing the second-order derivative matrix of the limit state surface and solving an associated eigenvalues problem.
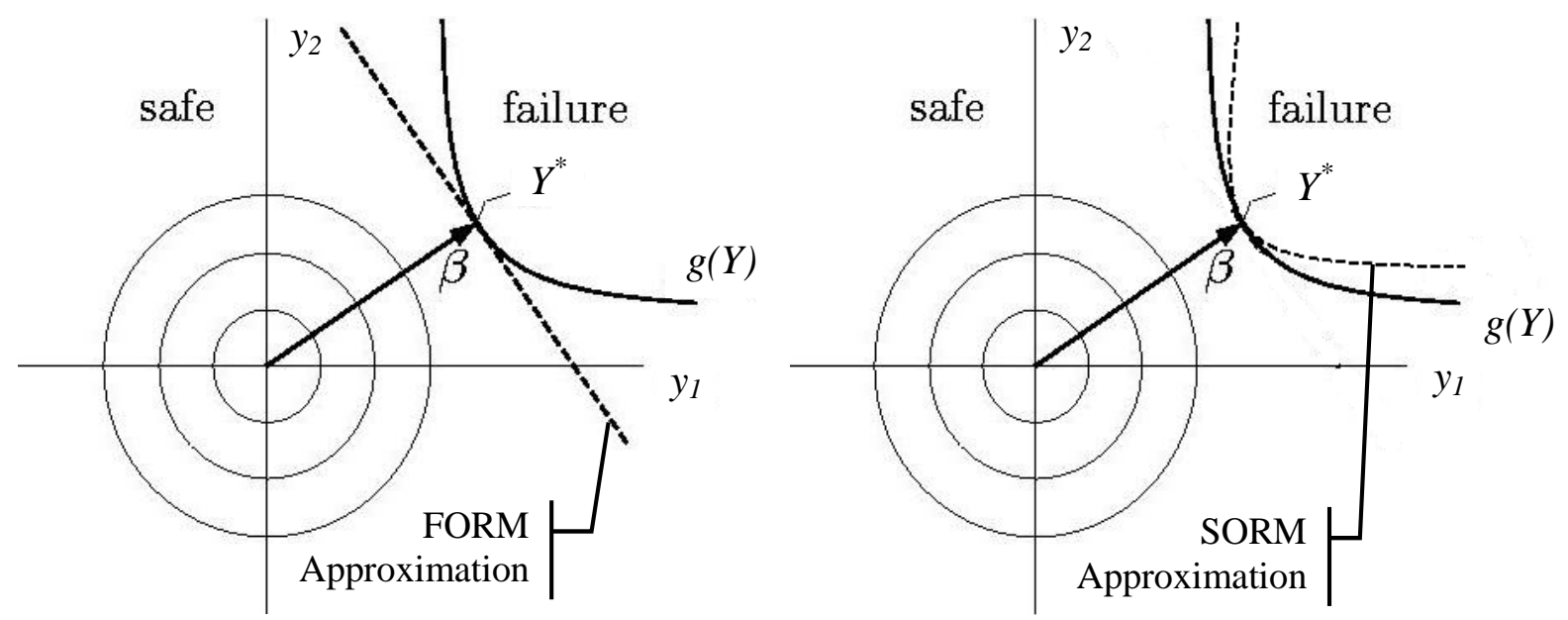

Figure 4: FORM and SORM approximations in standard normal space $Y$

The determination of the MPP involves the solution of the following constrained optimization problem:

$$
\left\{\begin{array}{l}
\min _{y}\left[f(y)=\frac{1}{2} y^{T} y\right] \\
\text { subject to } G(y)=0
\end{array} \text { with } y \in Y\right.
$$

Many algorithms are available in literature to solve this problem, usually combining a direction search algorithm and a line search procedure along that direction. A well-established algorithm for the reliability index approach (RIA) is the modified HL-RF [5] algorithm, and for the Performance Measure Approach (PMA), the Hybrid Mean Value (HMV) scheme [11] exhibits good performance. 


\section{E. Limitations of LSA}

Although they are well established and widely used, LSA approximations such as the First Order Reliability Method (FORM) and the Second Order Reliability Method (SORM) exhibit some limitations when the degree of nonlinearity in the LSF increases. Since the FORM approach is a linear approximation, it is easy to understand that it ignores all nonlinearities in the LSF. As a result, it only relies on the estimated reliability index $\beta$. Also, note that if the LSF is defined as a series of failure criteria (union of failure domains) or as parallel systems (intersection of failure domains) special extensions should be considered [4][5]. Furthermore, the SORM approximation needs the evaluation of the main curvatures, which makes it not suitable for high dimensional problems and multiple failure domains [15].

As the number of dimensions of the problem increases, also the approximation error for all Limit State Approximations increases accordingly, without the possibility to estimate this error. This means that for problems with a number of dimensions $n>15$, special care should be paid to the degree of nonlinearity of the performance function(s) because the error of the approximation strongly depends on it and cannot be known nor estimated [15].

However, an upper bound to the probability of failure estimation can always be calculated. Recalling that $\beta$ measures the distance from the origin of the standard normal space to the point on the limit state function closest to the origin, then an upper bound on $P_{f}$ is given by the probability content outside a (hyper-)spherical limit state function with radius $\beta$, centered at the origin of the $Y$ space:

$$
g(y)=\beta^{2}-\sum_{i=1}^{n} y_{i}^{2}=0
$$

Since the $Y_{i}$ are normally distributed and independent, $\sum_{i=1}^{n} y_{i}^{2}$ has a chi-squared distribution $\chi_{n}$ with $n$ degrees

of freedom. The probability of failure is then $P_{f}=1-\chi_{n}\left(\beta^{2}\right)$. When $n=1$ the probability content is twice that given by a single linear limit state surface [4].

For large dimension analyses $(n>30)$, also the performance of gradient-based algorithms (as used in the HL-RF algorithm and in PMA procedures) decreases with the increasing problem dimension. Furthermore the computation time of finite difference gradients for high dimensional problems also depends on the increased number of points that are needed to accurately model the non-linearity [14]. 
Finally, the necessity to compute the position of the MPP using a gradient-based algorithm does not guarantee that the obtained point is a global minimum, especially when non-normal distributions are used. Therefore, special procedures are needed to deal with nonlinearities, which further increase the computational effort that is required.

\section{F. Considerations on using the Finite Element Method}

All the algorithms used, however, don't consider if the point they are searching for has a physical meaning or not. They only solve the mathematical problem, thus some restrictions should be enforced to avoid that the MPP is searched in the region of the transformed space where the results have no physical meaning (although they are mathematically possible). In this paper the word "physical" refers to the used FE model and to the particular characterization of the variability of the input parameters, including their boundaries. In fact, when using FE models to represent reality, at least three processes should be carried out, as stated by the Technical Committee on Model Credibility of the Society for Computer Simulation in 1979 [16]. This process, shown in Figure 5, identifies the primary phases and activities of modeling and simulation, including model Verification and Validation (V\&V).

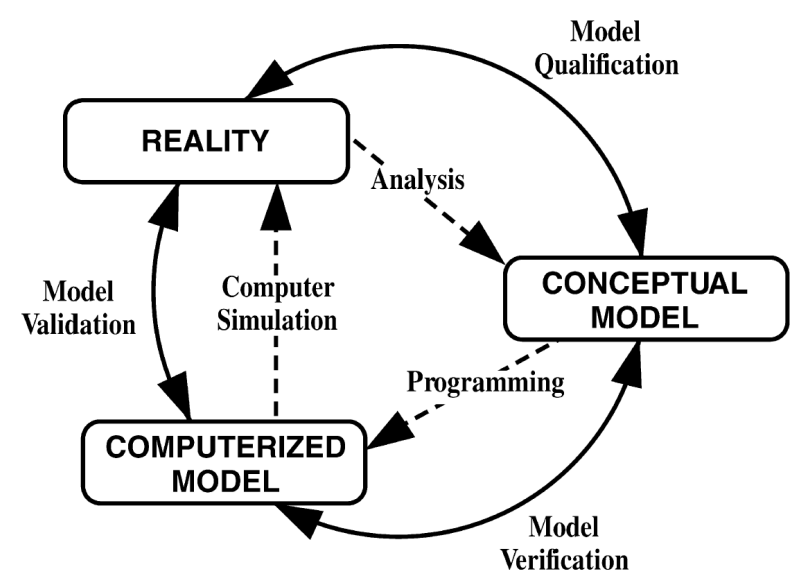

(C) 1979 by Simulation Councils, Inc.

Figure 5: View of modeling and simulation by the Society for Computer Simulation

As defined by the AIAA Verification and Validation Guide [17], Verification is the process of determining that a model implementation accurately represents the developer's conceptual description of the model and the solution to the model, while Validation is the process of determining the degree to which a model is an accurate representation of the real world from the perspective of the intended uses of the model. These definitions imply that the FE model 
cannot be used with any possible combination of the identified input parameters, disregarding the domain of verification and validation of the model itself. In this view, given a verified and validated FE model, three sets of input parameter combinations can be identified:

- Set 1: combinations of input parameters, fully included in the verified and validated domain, that yield accurate analysis results. Combinations included in this set well represent reality. The analysis of the models using these combinations yields results that have a high degree of credibility.

- Set 2: combinations of input parameters that are on or outside the boundary of the verified and validated domain. These combinations are still physically meaningful but can yield inaccurate analysis results. In fact, depending on the model, some results can still be valid and usable but their credibility is not assessed because the used combination of input parameters is on the boundary of the verified and validated domain. For example, when a structural failure occurs (intended as a violation of the performance criterion), even if the combination of the input parameters has a physical meaning, the information given by the analysis results has a low degree of credibility since there is no usable information on the actual magnitude of the stress/strain field that produced the failure. The main reason for this behavior is that FE solvers are usually not able to predict fractures and their consequent effects on the structure with conventional models.

- Set 3: combinations of input parameters that are outside the verified and validated domain and that are physically meaningless. These combinations usually yield no results, since the constitutional laws used to build the conceptual model (see Figure 5) are generally violated or not valid anymore, thus invalidating also the computerized model.

The latter case seems very simple but has some implications. In fact, the points where FE computations are not possible at all represent a boundary to the stochastic domain. For the reliability problem, these boundaries mathematically cut the tails of the joint normal distribution in the standard normal space of the integrals of Eq. (1) and (2), thus explicitly assigning a zero probability (impossible event) to the excluded domain. This means that the effective stochastic space (having total probability equal to 1) is limited to the region of possible computations with a high or sufficient degree of credibility (input parameters combinations of Sets 1 and 2). No mathematical algorithm should search the MPP outside that region.

Unfortunately the boundaries of the domain of possible computations are unknown or extremely difficult to be assessed correctly. The assumption of an unbounded stochastic space, which till now has always been accepted in 
literature, is typically not valid for FE computations. Also, there is an unknown error in the $P_{f}$ estimation that is not due to the specific approximation of the limit state surface in the MPP (FORM or SORM), but due to the definition of the domain itself, especially when using non-gaussian random variables.

In this paper the above-mentioned problem has been simplified for engineering practice: the input parameters have been bounded inside a hypercube centered in the origin with an edge size varying from 10 to $30 \sigma$, depending on the values of the input parameters scatter. This avoids feeding the FE solver with Set 3 combinations of input parameters and keeps the algorithm inside the region that can be explored by the Finite Element method with a verified and validated model. For example, in the case of a $20 \sigma$ edge hypercube, the nearest boundary is located at $10 \sigma$ from the origin, which corresponds to a failure probability of less than $2.0 \cdot 10^{-23}$. It could also be the case that the nearest point lays outside the hypercube of $20 \sigma$; in that case, it is guaranteed that the reliability index is higher than $10 \sigma$, which is sufficient for all practical purposes as quality control processes could reject components with a deviation from a nominal value greater than $10 \sigma$.

\section{Analysis Case Implementation}

With the variability introduced in the input parameters, a first estimation of the reliability of the nominal design is made. This allows the estimation of the degree of conservatism for the nominal design and how much it can be improved without violating the target reliability level. Then the performance behavior of the FE model has been observed and an RBDO process has been applied to optimize the design by reducing the total mass while satisfying the following probabilistic constraint:

$$
\beta_{t}=6 \Rightarrow P_{f}=9.86 \cdot 10^{-10}
$$

In order to perform the RBDO procedure, a software code has been developed and used. The optimization process is managed by an OPTIMUS [18] thread that determines the necessary steps to reach the optimum design point that satisfies the probabilistic constraint of Eq. (16), using a Sequential Quadratic Programming (SQP) approach. Note that the optimization process is a deterministic process with a probabilistic constraint. Thus the result is an optimum point that is also robust with respect to the required failure probability.

For each iteration of the optimization process, the algorithm requires the evaluation of the performance function at specific values of the input parameters, so that a static FE analysis has to be performed for each evaluation. To 
accomplish this task, another process management thread has been created that submits the new problem to the FE solver and extracts the necessary results.

The fatigue analysis of the slat track requires the integration of different procedures. Given a material degradation model, a standard durability assessment usually requires one structural analysis with unit loads together with the simulation of the repetition of the selected load history for a number of cycles. Further details will be given in Section IV.B.

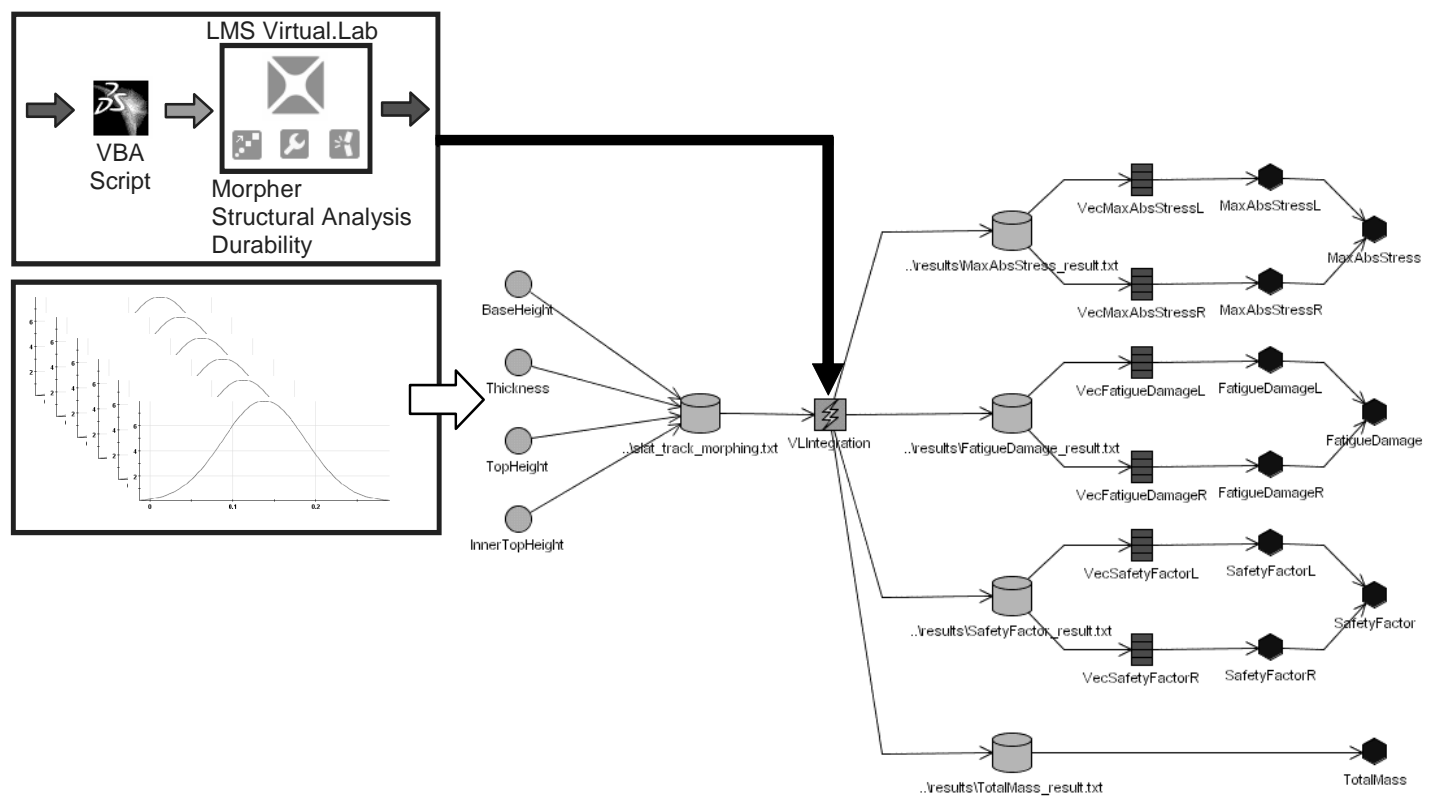

Figure 6: Process integration graph

For the present case, another step is required before the structural analysis can be performed. In fact, to take into account the variability of the input parameters, a transformation of the mesh is required. This transformation turns the nominal mesh into a morphed mesh, for which the values of the geometric input parameters are different from those of the nominal design. This morphed mesh can better represent the real product, without the necessity of remeshing from the CAD geometry. Thus, the slat track mesh first needs to be modified to reflect the new input parameter configuration and then analyzed with unit loads using a FE solver. The results of the structural analysis are then used for the durability simulation. The outcome of the fatigue analysis, in terms of safety factor, can finally be used as the performance function for the slat track. 


\section{A. Mesh Morphing}

This type of mesh transformation, called mesh morphing [19], consists of a change in the position of the nodes belonging to a specified mesh, following a given expression. This expression can be mathematical, such as a matrix, or geometrical, for example the definition of a target shape. The morphing technology offers very interesting perspectives to apply such mesh modifications in a user-friendly and application-oriented way [20]. The most commonly used mesh morphing techniques are free form morphing and control based approach.

In free form morphing, a set of morphing tools acts directly on the mesh. The approach uses the concept of control nodes, deformable nodes and fixed nodes. Fixed nodes determine the boundary of the area of the deformable zone of the mesh. The deformable nodes correspond to the nodes that will be morphed. The actual displacement of the deformable nodes is determined by the displacement of the control nodes. To move the control nodes, several transformations can be applied including projection (to surfaces or lines), translation, rotation, alignment and scaling.

In a control based approach, morphing operations are not carried out directly on the mesh of the existing FE model. Instead, morphing is applied by means of control blocks. Each control block represents a volume that envelops a small part of the existing FE mesh. By linking the location of the FE nodes inside each control block to the location of the corners of the control block, morphing operations applied to the blocks automatically define the geometry of the morphed FE model.

For the case considered in this paper, the first approach has been selected. The particular complexity of the mesh made the use of control blocks less suitable. In particular, referring to Figure 1 and Figure 2, four different free form morphing set-ups have been created, one for each input parameter. Each set-up has a set of control nodes, deformable nodes and fixed nodes and can share nodes with other set-ups, thus allowing overlapping (or cumulative) transformations. For this reason, a sequence of application of the 4 transformations has been defined to reduce to the minimum the possible distortion of the geometry of the finite elements involved in the transformation.

The morphing process has to be repeated a number of times for each combination of the input parameters. The performance function needs to be evaluated for each set of mesh morphing transformations, thus requiring each time a durability analysis. 


\section{B. Durability analysis}

It is well known that fatigue is the degradation of a material due to repeated cyclic loading. For metals, this typically means that there is an initiation of small cracks from active slip bands in grains on the free surface of a specimen, component or structure. These small cracks eventually link to form large cracks that either break or severely degrade the performance of a component.

Two basic approaches have been developed to estimate the crack initiation life of components and to address fatigue analysis and design for durability: the stress-life approach and the strain-life approach. The goal of both approaches is to estimate the crack initiation life of structures by combining a mechanics-based analysis of stresses or strains with the results of basic material property tests. The strain-life approach was developed in the early 1960's as an alternative to the stress-life approach for determining fatigue damage, particularly when yielding occurs at critical locations of a component. The basic idea of the strain-life approach is that on most useful engineering components, locations of stress concentration, or notches exist at which the local stress state may be above the yield stress of the material, even if most of the surrounding material remains elastic.

The fact that the location where yielding has occurred is surrounded by material still in elastic state, restricts the deformation of the yielded material. This results in an elastic-plastic deformation mechanism at the notch location. The notches, in turn, serve as critical locations for fatigue cracks to initiate because of the higher stresses and strains at these locations. Therefore, the fatigue behavior of a material can be related to strain-controlled material property tests.

When repeated fatigue life tests are performed, one does not find exactly repeated results, but rather a scattered set of results. There are natural variations in material properties, component dimensions, customer service loads and manufacturing tolerances. Probabilistic distributions can be used to characterize the variation of these variables and include this variability in the design process. This better explains and predicts the scatter obtained in fatigue test results.

For the slat track, in-flight measured data has been used for the fatigue analysis [21]. The aircraft has been instrumented as for the normal qualification test campaign, where several different measurements are usually acquired in many different flight conditions. From this large database, a subset of measuring channels has been selected to retrieve the measurements of interest for the fatigue assessment on the slat-track. All significant flight 
conditions have been identified on the selected channels and the necessary data has been extracted. This represents a reliable base for the fatigue life prediction.

In particular, all loads acting on the slat track were measured at three locations (Figure 7). The acquisition and recording of the bending moment $M_{B}$ and of the axial beam force $L$ was done by using strain gauges at these locations. Also, the moment $M_{P}$ of the pinion acting on a rack at the inner side of the slat track was measured at different positions, depending on the slat-setting angle $\gamma_{S S}$.

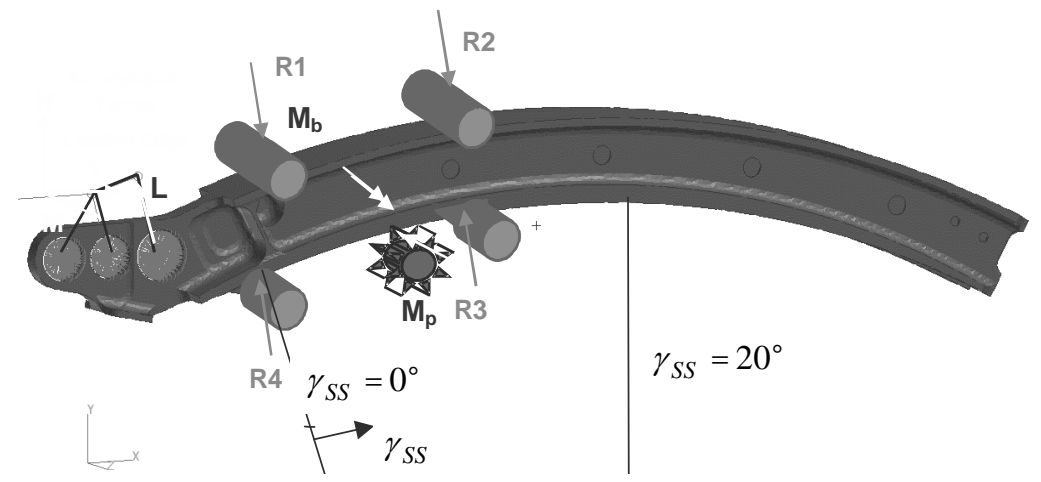

Figure 7: Kinematics boundary conditions for varying slat track position $\gamma_{S S}$.

Under operating conditions, the slat track is a moving component whose position can be characterized by the slat-setting angle $\gamma_{S S}$. A dedicated motor that drives a pinion mechanism actuates the slat motion. Since test data has been acquired in different flight conditions, measured loads refer to different slat extensions. As a consequence, loads need to be applied at different positions for an accurate numerical simulation based on unit load cases.

Taking into account such load conditions, a number of different kinematics boundary conditions for the different slat track positions have been simulated. These cover the total range of slat track positions between the fully retracted position $\left(\gamma_{S S}=0^{\circ}\right)$ and the fully extended position $\left(\gamma_{S S}=20^{\circ}\right)$. Transition matrices have to be determined for all different setting angles of the slat to compute the aerodynamic forces $T, N$ and $M$ at the leading edge of the slat.

Kinematics boundary conditions are defined by the varying position of the rollers (R1-R4 in Figure 7), which change relative location simultaneously with the measured load $M_{P}$. The rollers constitute a set of kinematics constraints, as radial displacements have to be zero at roller locations while the pinion carries transversal loads. The 
group of active forces is however acting in the symmetry plane $z=0$, therefore all in-plain reaction forces are taken in consideration with the defined boundary conditions.

The unit load solution for $N=1 \mathrm{~N}$ has been computed for the setting angle $\gamma_{S S}=20^{\circ}$. In fact, noting that for $\gamma_{S S}>7^{\circ}$ all rollers are out of the $M_{B}$ cross section (see Figure 7), it can be assumed that the transition matrix between measured loads and aerodynamic loads at the leading edge is nearly constant for the whole range of conditions between $7^{\circ}$ and $28^{\circ}$ that are included in the measured data. Therefore, the unit load case simulated for $\gamma_{S S}=20^{\circ}$ represents the most critical condition. An extensive analysis of the fatigue performance of the slat track is reported in [21].

For an efficient stress analysis at the boundaries of a three-dimensional component, an extremely thin skin of shell elements on the surface has been used to build up an evaluation group. This drastically reduces the result file size and saves computation time for fatigue evaluation. For the slat track model, 76344 TRIA3 elements of thickness $0.001 \mathrm{~mm}$ have been generated on the surface. These elements do not affect the stiffness matrix and they are only used for data recovery.

For the present case, the strain-life approach has been selected and the cyclic stress-strain curve and the strainlife curve have been determined using the Uniform Material Law (UML) [22]. Further details on the validation of the UML model can be found in [21].

The durability performance of the slat track has been expressed in terms of a safety factor. The safety factor is always defined as the factor that can be applied to the loads in order to reach the endurance limit (infinite life) with respect to the amplitude of the largest stress cycle. For example, a safety factor of 2 means that the load does not cause any cyclic damage accumulation as long as it is scaled with a factor $<2$. Damage accumulation for high or low cycle fatigue failure starts when the factor is $>2$.

In this paper the minimum safety factor on the FE model has been considered as representative of the fatigue performance of the slat track. The element where the minimum safety factor is computed will be called "hot spot" and can change depending on the variation of the input parameters. Further details are given in Section V.A.

\section{Process Integration}

In order to automate the whole procedure, a process integration set-up has been created. The integration has been divided in two steps: integration of the mesh morphing/durability analysis and the creation of the necessary input 
and output files needed for the analysis. The first part was accomplished by using LMS Virtual.Lab [23] together with a Visual Basic Application script. This block has been integrated in an OPTIMUS [16] graph, providing the necessary problem definition, input/output files, results extraction and post-processing (see Figure 6).

Since the computational effort for the whole process can be considerable for industrial FE models, a Design Of Experiments (DOE) [24] approach has been selected to limit the time needed. A Latin Hypercube DOE in the range of $[-15 \sigma,+15 \sigma]$ has been used to compute the performance of the structure in 80 points. This range has been chosen to allow a sufficiently wide range of exploration of the response of the structure in the standard normal space. Subsequently, a least squares Response Surface (RS) [25] has been created to approximate and predict the response of the structure without requiring a full analysis.

The use of Response Surfaces can be however prone to errors, especially for reliability problems [25]. In fact, in this type of analysis the correct approximation of the limit state function in the stochastic space is essential. Even a small error in the estimation can cause a considerable variation in the approximation of the probability of failure due to the non linear relation between the reliability index $\beta$ and the standard normal CDF $\Phi$ (see Eq. (5)). Thus a careful check of the validity of the models has been carried out (see Section V.A).

\section{Performance function selection}

Following the previous considerations, the choice of the performance function $G$ for the reliability analysis and optimization is related to the desired Safety Factor minimum. For the slat track, a Safety Factor minimum value of 2.5 has been selected. The performance function can then be written as

$$
\text { SafetyFactor }=2.5 \Rightarrow G=\text { SafetyFactor }-2.5
$$

With this choice, the $G$ function is positive where the Safety Factor is larger than 2.5.

The threshold value of 2.5 for the Safety Factor has been selected because it is close to the minimum value for the safety factor observed during the DOE, which constitutes the domain of validity of the RSM. In the case that a wider sampling interval for the DOE is possible, the quality of the RSM can be improved, thus allowing the selection of a lower threshold.

The probabilistic constraint of Eq. (5) and (16) can thus be written

$$
P(G<0)=P(\text { SafetyFactor }<2.5)=9.86 \cdot 10^{-10}
$$




\section{Results}

\section{A. Results of the Design Exploration}

The results of the design exploration are reported here. The range of exploration of the system response has been $[-15 \sigma,+15 \sigma]$ for each input parameter and a Latin Hypercube DOE strategy has been selected. This strategy is a similar to random sampling with the difference that the $\mathrm{N}$ samples placed in the $\mathrm{n}$-dimensional space are placed in subsets of equal probability, thus improving the efficiency of the sampling distribution. The results have been reported in terms of Safety factor and Total Mass and can be seen in Figure 8 and Figure 9.
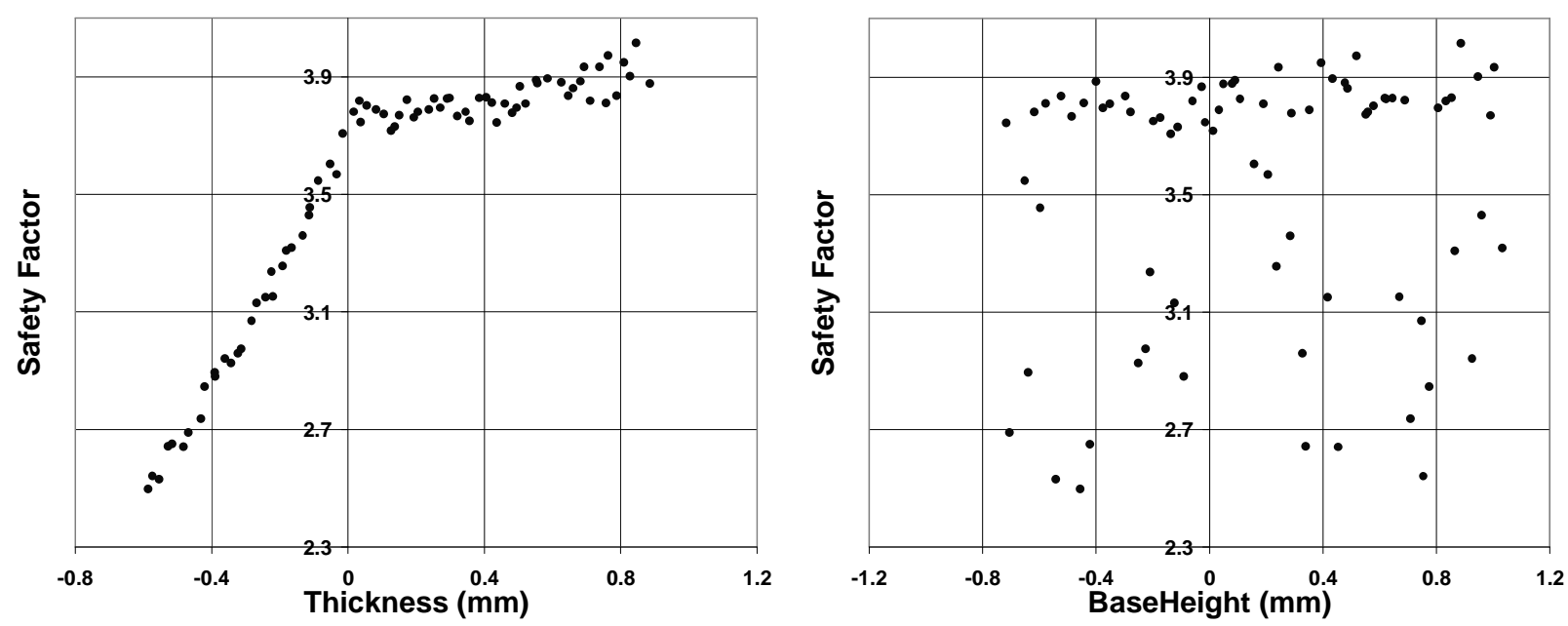

Figure 8: Results of the Latin Hypercube DOE for the Safety Factor vs. the Thickness and the Base Height parameters.
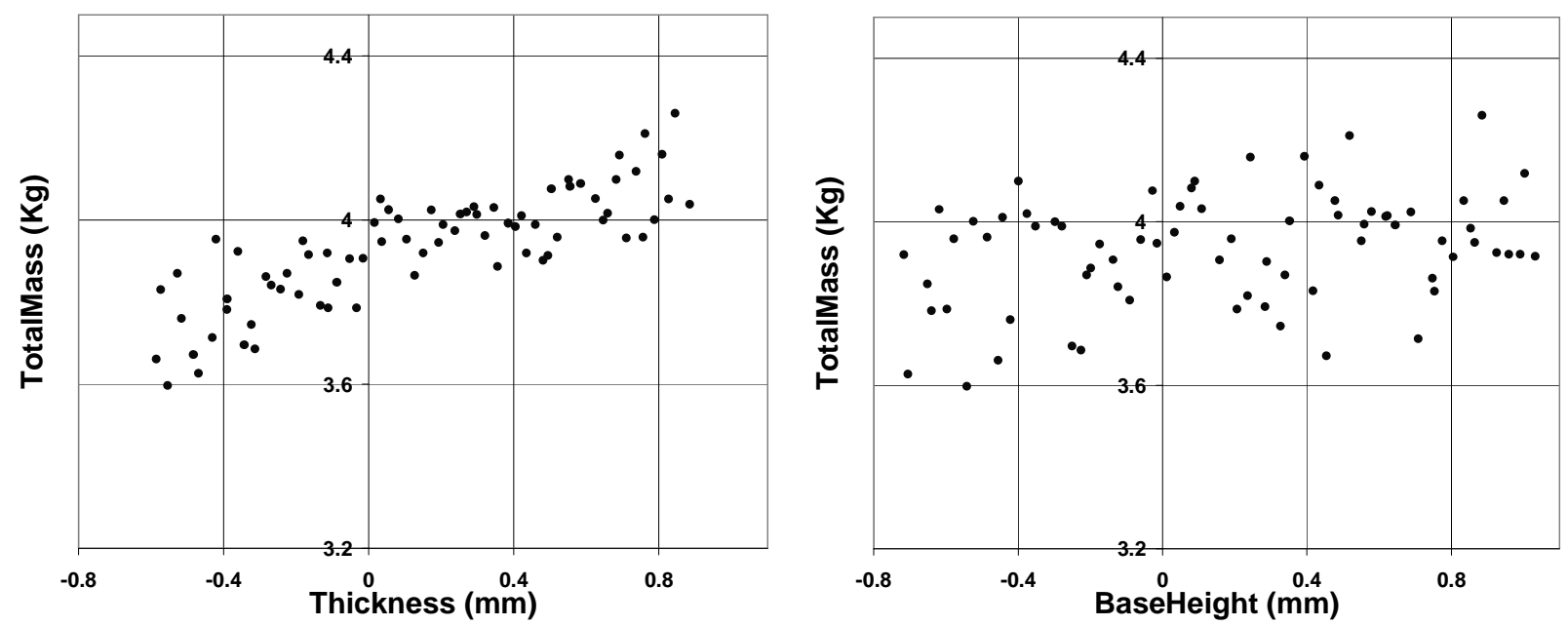

Figure 9: Results of the Latin Hypercube DOE for the TotalMass vs. the Thickness and the BaseHeight parameters. 
It can be seen from Figure 8 that the relation between the safety factor and the input parameters is not the same for all of them. Particularly, a variation of the Thickness parameter (defined as the difference between the actual and the nominal value) determines two different samples populations in the safety factor. In fact, if the Thickness parameter is negative, the approximate slope of the population is steeper than the case where the Thickness parameter is positive. This shows that the sensitivity coefficient of the safety factor w.r.t. a variation of the Thickness parameter changes considerably, depending on the values of the Thickness. Moreover, the scatter of the two populations due to the variation of the other parameters is small. This means that the dependency between the Thickness parameter and the Safety Factor is not significantly influenced by the variation of other parameters.

To better explain the behavior of the system response showed in Figure 8, a careful analysis of the values of Safety Factor on the FE model has been done. In particular, the identification of the elements where the Safety Factor assumes the minimum values (also called "hot spots") has been carried out. Since the performance of the system is represented by the minimum value of the Safety Factor, the location of the first hot spot also identifies the element of the FE mesh where this minimal value occurs. The analysis revealed that the first hot spot where the Safety Factor is minimal changes depending on the value of the Thickness, as defined in Section II.A. The different locations of the first hot spots for the cases of negative and positive Thickness difference from the nominal value are showed in Figure 10 and Figure 11.

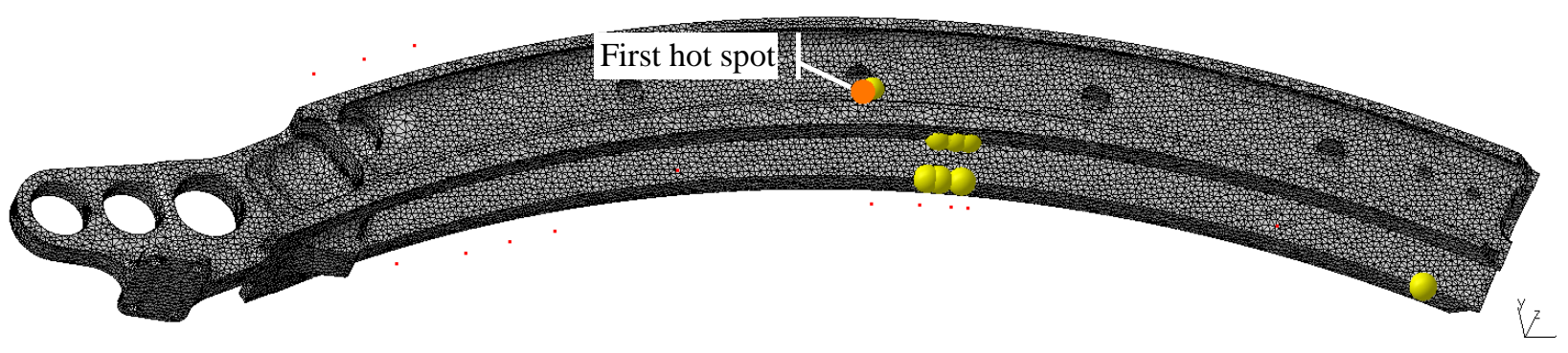

Figure 10: Location of the first hot spot for a negative Thickness difference 


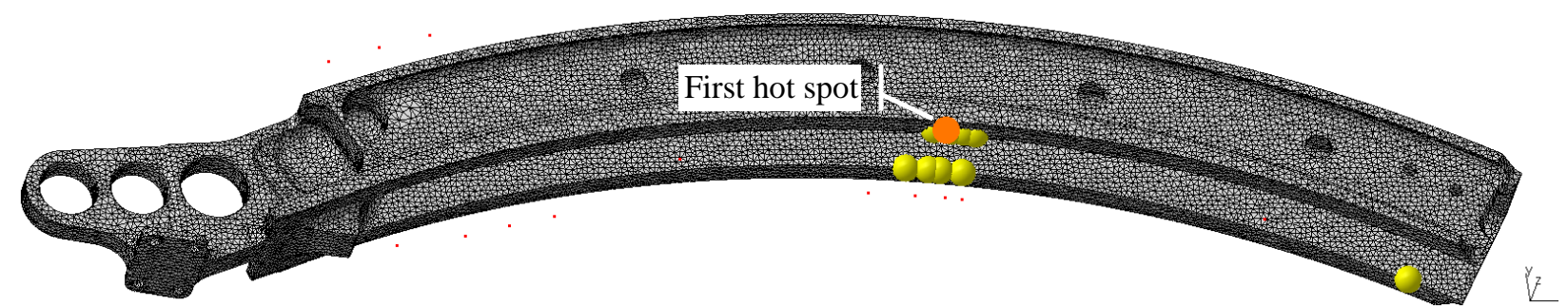

Figure 11: Location of the first hot spot for a positive Thickness difference

In fact, when the Thickness difference is positive, the predominant hot spot location (for the first and the other hot spots) is the one showed in Figure 11. When the Thickness difference decreases, a new predominant location can be identified that is more sensible than the first one to variations of the Thickness parameter. This new location is showed in Figure 10. This explains why the scatter plot of Figure 8 shows a population of samples with two different linear behaviors. This has been taken into account in the Response Surface calculation process, as explained later.

On the other hand, the behavior for the Total Mass is smoother than the Safety Factor and for all the parameters there is only a small linear correlation. This is logical, as mass depends linearly on geometrical dimensions.

The results of the DOE have been used to build two response surfaces, one for the Safety Factor and one for the Total Mass. In order to model with sufficient accuracy the dependency of the Safety Factor from the Thickness parameters, an Optimal RSM (O-RSM) [26][27] procedure has been carried out to find out an "optimal" selection of terms for a least squares model within the DOE domain of exploration of the input parameters. In this procedure, for a given set of simulated data pairs $\left(x^{i}, y^{i}\right)$ with $i=1 \ldots N, x^{i} \in \mathfrak{R}^{n}$, an iterative process is carried out to find the basis $B_{j}(x)$, with $j=1 \ldots m$, of functions defined in $\mathfrak{R}^{n} \rightarrow \mathfrak{R}$ and the set of coefficients $\alpha_{1}, \ldots, \alpha_{m}$ that, given the linear fitting function

$$
y\left(x ; \alpha_{1}, \ldots, \alpha_{m}\right)=\sum_{j=1}^{m} \alpha_{j} B_{j}(x)
$$

minimizes the sum

$$
\sum_{i=1}^{N}\left[y^{i}-y\left(x^{i} ; \alpha_{1}, \ldots, \alpha_{m}\right)\right]^{2}
$$

For the Safety factor, the selected basis $B_{j}(x)$ of functions was a third order polynomial with first order sine and cosine functions 


$$
\begin{aligned}
B(x)= & \{1\} \cup \\
& \left\{x_{i_{1}} \mid i_{1}=1, \ldots, n\right\} \cup \\
& \left\{x_{i_{1}} x_{i_{2}} \mid i_{1}, i_{2}=1, \ldots, n \quad i_{1} \leq i_{2}\right\} \cup \\
& \left\{x_{i_{1}} x_{i_{2}} x_{i_{3}} \mid i_{1}, i_{2}, i_{3}=1, \ldots, n \quad i_{1} \leq i_{2} \leq i_{3}\right\} \cup \\
& \left\{\sin \left(x_{i_{1}}\right) \mid i_{1}=1, \ldots, n\right\} \cup\left\{\cos \left(x_{i_{1}}\right) \mid i_{1}=1, \ldots, n\right\}
\end{aligned}
$$

The resulting fitting function that used this basis showed to be more suitable to efficiently model the special correlation between the Thickness parameter and the Safety factor, while keeping a good accuracy also for the other parameters. Some sections of the model are shown in Figure 12.

For the Total Mass, the selected response model was a quadratic Taylor polynomial with a strong prevalence of linear terms over quadratic terms, which well represents the computed samples (see Figure 13).
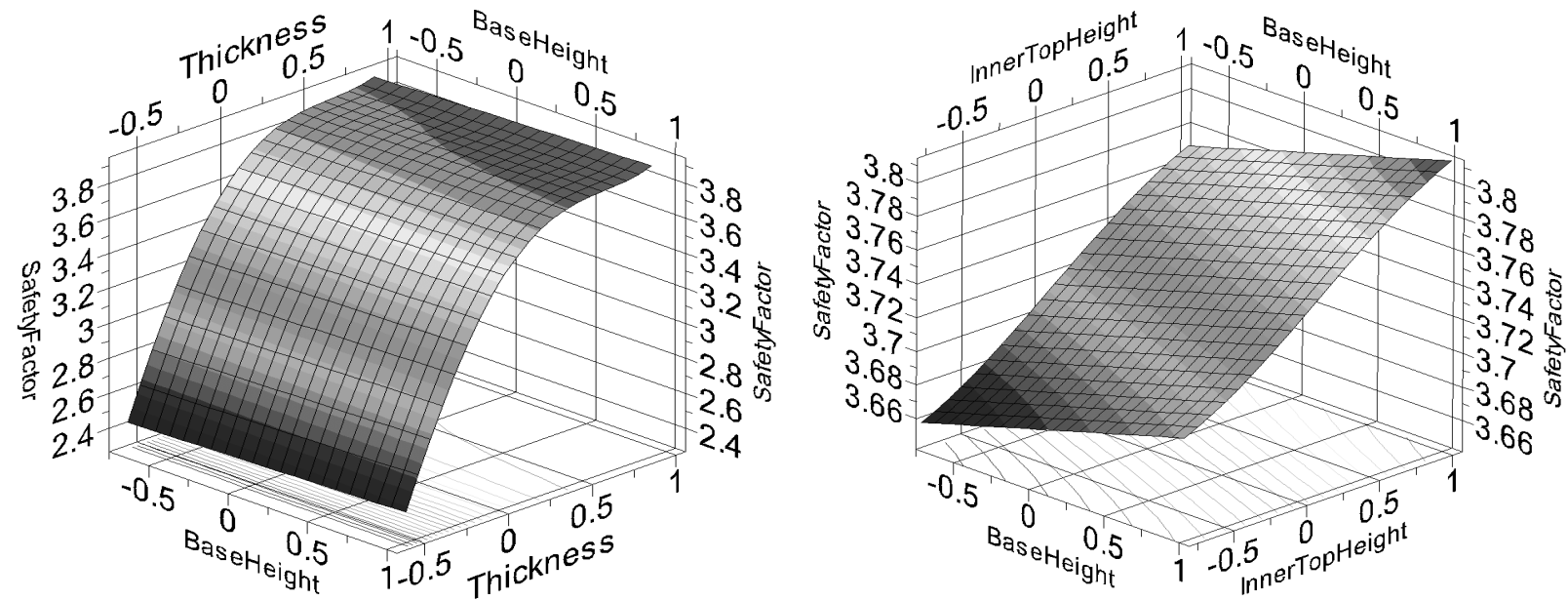

Figure 12: Sections of Response Model for the Safety factor.
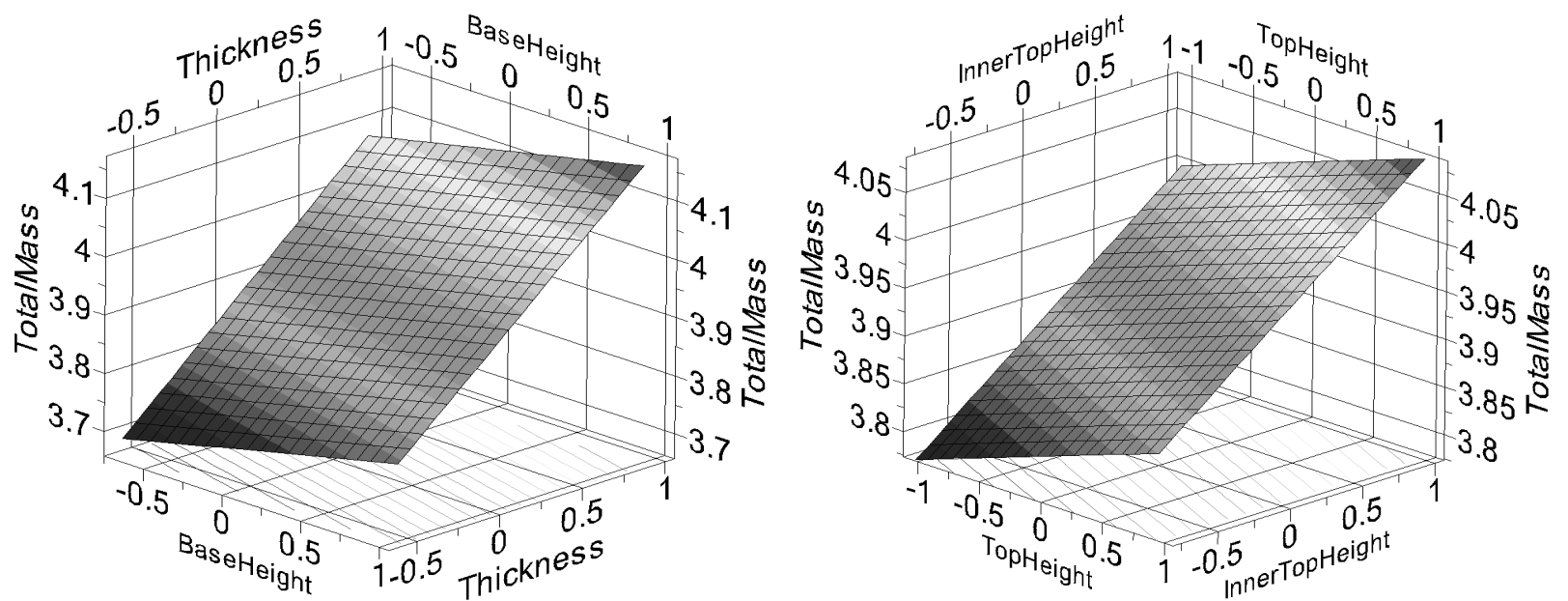

Figure 13: Sections of the Response Model for the Total Mass. 
For each response model, quality indexes have been computed to verify the fitting and prediction accuracy within the limits of the domain of definition of the models. In particular, three regression parameters have been computed: $R^{2}, R_{a d j}^{2}$ and $R_{\text {press. }}^{2}$

The standard regression parameter $R^{2}$ is defined as

$$
R^{2}=1-\frac{\sum_{i=1}^{N}\left(x_{i}-\tilde{x}_{i}\right)^{2}}{\sum_{i=1}^{N}\left(x_{i}-\bar{x}_{i}\right)^{2}}
$$

where $x_{i}$ is the simulated result, $\tilde{x}_{i}$ is the result predicted by the RSM and $\bar{x}_{i}$ is the mean of all simulated results.

A value of $R^{2}$ close to 1 means that the model well fits the values of the simulation points used to calculate the model.

The adjusted regression parameter $R_{a d j}^{2}$ is defined as

$$
R_{a d j}^{2}=1-\frac{\sum_{i=1}^{N}\left(x_{i}-\tilde{x}_{i}\right)^{2}}{\sum_{i=1}^{N}\left(x_{i}-\bar{x}_{i}\right)^{2}} \cdot \frac{n-1}{n-m}
$$

This adjusted regression value takes into account the number of experiments $n$ and the number of terms $m$ in the model. The higher the number of terms in the model, the lower the value of $R_{a d j}^{2}$. Of course, if a sufficient number of terms is available, the model will more easily follow the simulated points, and therefore models with a high number of terms are penalized. $R_{a d j}^{2}$ is generally smaller than $R^{2}$. Again, a value of $R^{2}{ }_{a d j}$ close to 1 means that the model well fits the values of the simulation points used to calculate the model.

The third parameter, $R_{\text {press }}^{2}$ indicates the predictive capability of the model. Basically it gives a measure of how good the model will predict values for points that were not simulated yet. To calculate this parameter, the first experiment is left out of the experiment list and a new model is calculated based on the $n-1$ remaining experiments. Then a check with this new model is made on how well it predicts the simulated value for this left-out experiment. This is repeated for each experiment, and the result of all this calculations is summarized in one regression value. Actually it is not needed to calculate all these models, since there is an equivalent formula based on the result of the singular value decomposition that is used to calculate the RSM. If the value of $R_{\text {press }}^{2}$ is close to 1 , it indicates that the model will perform well for points that were not simulated. 
The quality indexes for the Safety Factor and the Total Mass models are reported in Table 3.

\begin{tabular}{|l|c|c|c|}
\cline { 2 - 4 } \multicolumn{1}{c|}{} & $\mathrm{R}^{2}$ & $\mathrm{R}_{\text {adj }}^{2}$ & $\mathrm{R}_{\text {press }}^{2}$ \\
\hline Safety Factor Model & 0.9860 & 0.9841 & 0.9806 \\
\hline Total Mass Model & 0.9999 & 0.9999 & 0.9999 \\
\hline
\end{tabular}

Table 3: Quality indexes of the Response Models for the Safety Factor and the Total Mass.

\section{B. Results of the Reliability Analysis}

Given the analysis case of Section II and the Reliability approach of Section III, a reliability analysis using the Reliability Index Approach (RIA) [10] has been carried out. The Response Models computed for the Safety Factor and the Total Mass have been used as meta-models in order to assess the reliability of the slat track structure.

The reliability analysis step is necessary to find the position of the Most Probable Point (MPP) on the LSF in the standard normal space [13]. The distance between the MPP location and the design origin in the standard normal space (reliability index $\beta$ ) represents a measure of the possible improvement on the design parameters that can be gained with the reliability-based optimization procedure (step 2) using a $6 \sigma$ target (see Section C).

The results of the reliability analysis procedure are reported in Table 4 and Table 5 and shown in Figure 14 . The starting point is the nominal design, characterized by the mean values of the input parameters.

\begin{tabular}{|l|r|}
\hline Number of Iterations & 7 \\
\hline Number of LSF evaluations & 52 \\
\hline Reliability Index $\beta_{s}$ & 14.398 \\
\hline
\end{tabular}

Table 4: Reliability analysis results of the nominal point using RIA

\begin{tabular}{|r|c|r|r|}
\hline Base Height & Thickness & Top Height & Inner Top Height \\
\hline$-1.3586 \sigma$ & $-14.324 \sigma$ & $-0.3000 \sigma$ & $-0.4330 \sigma$ \\
\hline $0.0714 \mathrm{~mm}$ & $-0.5639 \mathrm{~mm}$ & $0.1856 \mathrm{~mm}$ & $0.0250 \mathrm{~mm}$ \\
\hline
\end{tabular}

Table 5: Location of the Most Probable Point in the standard normal space ( $\sigma$-coordinate) and in the parameters space. 

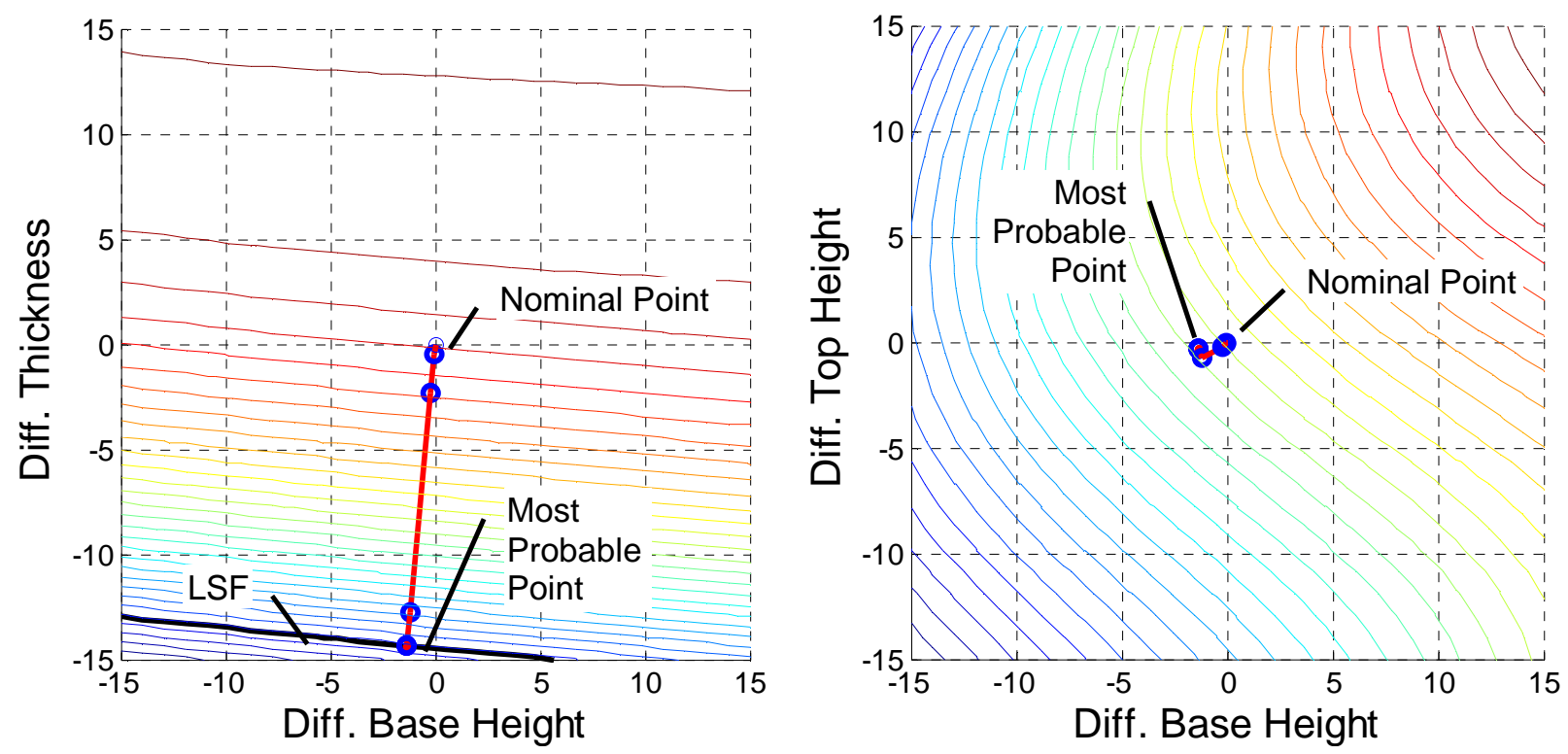

Figure 14: Results of the Reliability Analysis procedure - Location of the Most Probable Point in the standard normal space.

To better explain the results of the MPP location in Table 5, a negative value in the standard normal space means that the mean value should be reduced by the amount specified in standard deviation units. Thus the value is relative to the origin of the standard normal space, which is the nominal point (mean values). Thus, the results expressed in the $X$ space ( $3^{\text {rd }}$ row of Table 5$)$ are the new mean values of the difference between the design values and the mean manufacturing values (see also Section II.A for the definition of the input parameters).

To assess the effect of the introduced variability on the system response, a probabilistic characterization of the Performance Function (see Section IV.D) for the original design point has been carried out. Using the response surface, a Monte Carlo simulation of 2.000 samples has been used to estimate the mean and the standard deviation. The population obtained has then been analyzed with a least squares procedure using a histogram representation to find the best probabilistic density model that represents the population distribution. It has been found that the response of the system follows an extreme type III of smallest values distribution (sometimes called also Weibull of the smallest values), as reported in Table 6 and Figure 15. This model has been verified with a $\chi^{2}$ hypothesis test [7], which checks that the selected model is representative of the data set used for the least squares procedure. 


\begin{tabular}{|c|c|c|c|}
\hline Mean & Std. Dev. & Skewness & Kurtosis \\
\hline 1.235 & 0.041 & -0.501 & 0.36 \\
\hline
\end{tabular}

Table 6: Mean, standard deviation and higher moments of the generated population for the Performance Function response

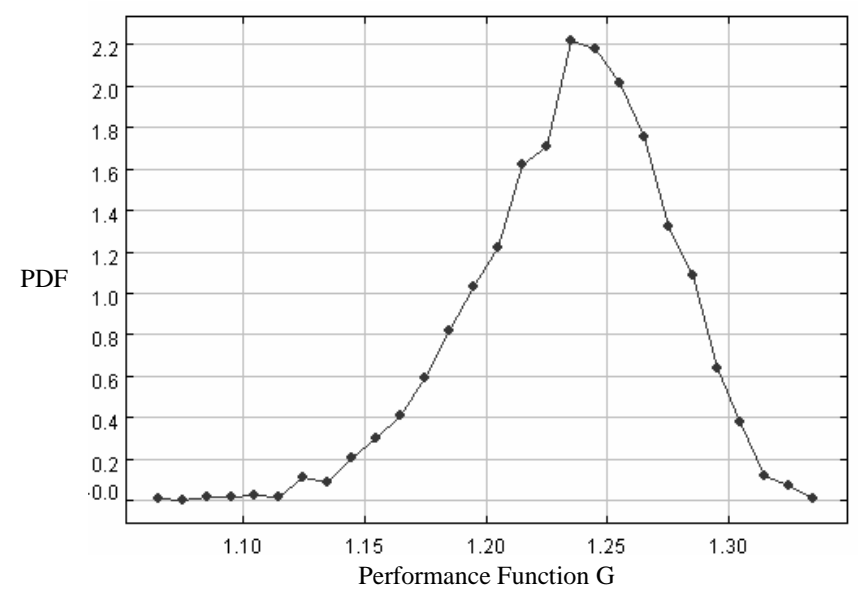

Figure 15: the Probability Density Function of the MCS population of the Performance Function $G$

\section{Results of the Reliability-based Design Optimization}

The results of the optimization process for the analysis case are reported in this section. The target of the optimization is to reduce the total mass, while keeping a distance of $6 \sigma$ from the region of the event space where the Safety Factor has a value equal or smaller than 2.5. This means that the newly found design will have, given the scatter in the input parameters, a probability of $9.86 \cdot 10^{-10}$ of having a Safety Factor smaller than 2.5 .

To carry out the RBDO process, a two steps strategy has been adopted. In the first step, a standard deterministic optimization procedure is performed to efficiently find a point located on the limit state function $G$ which minimizes the total mass of the structure. At this stage, no probabilistic constraint is taken into account and the point found has a probability of failure of approximately 0.5 (see Figure 17). In the second step, another optimization procedure is started from the deterministic optimum point to find the probabilistic optimum point, which satisfies the probabilistic constraint.

An alternative one step procedure can also be used. In this approach, the probabilistic constraint is enforced since the first optimization step. As a consequence, the probability of failure for each step of the optimization procedure has to be assessed in order to check that the probabilistic constraint is not violated. It is clear that, in some situations, this methodology could require a much higher number of computations w.r.t. the two step approach, which is 
usually undesirable and computationally expensive. In particular, experience showed that the 2 step approach is particularly useful when the MPP for the nominal design is located at a distance $\beta_{s}>1.5 \cdot \beta_{t}$ (e.g. the target reliability index $\beta_{t}$ is $6 \sigma$ and the reliability index of the current nominal design $\beta_{s}$ is $12 \sigma$ ). In this case, in fact, the optimization iterations that would require the evaluation of the probabilistic constraint would be limited to the $2^{\text {nd }}$ part of the optimization that pulls the optimum point to a distance of $\beta_{t}=6 \sigma$ (for the present case) from the deterministic one and that usually requires less iteration steps. This is demonstrated in Figure 18 and Figure 19.

In this case, in fact, if the probabilistic constraint would have been considered in only one single optimization procedure (instead of 2) starting from the nominal point, the number of evaluations required to enforce the constraint would require a much high number of performance function evaluations This approach constitutes an acceptable compromise between performance and accuracy.

For both optimization procedures, each input parameter has been constrained to a range of $[-15 \sigma,+15 \sigma]$, which is also the range of validity of the RSM and avoids extrapolation.

The results of the first step, deterministic optimization, are reported in Table 7 and Table 8 and shown in Figure 16.

\begin{tabular}{|c|r|r|r|r|r|r|}
\hline SQP Iteration & $\begin{array}{c}\text { Base Height } \\
(\mathrm{mm})\end{array}$ & $\begin{array}{c}\text { Thickness } \\
(\mathrm{mm})\end{array}$ & $\begin{array}{c}\text { Top Height } \\
(\mathrm{mm})\end{array}$ & $\begin{array}{r}\text { Inner Top Height } \\
(\mathrm{mm})\end{array}$ & $\begin{array}{r}\text { Total Mass } \\
(\mathrm{kg})\end{array}$ & $\begin{array}{r}\text { Performance } \\
\text { Function G }\end{array}$ \\
\hline Start & 0.151813 & 0.147218 & 0.025027 & 0.102719 & 3.9327 & 1.2373 \\
\hline 1 & 0.139451 & 0.111024 & 0.012170 & 0.076979 & 3.9188 & 1.2051 \\
\hline 2 & 0.077545 & -0.070060 & -0.052953 & -0.051852 & 3.8493 & 0.9892 \\
\hline 3 & -0.109533 & -0.594103 & -0.249716 & -0.440344 & 3.6443 & -0.1369 \\
\hline 4 & -0.109387 & -0.542136 & -0.247893 & -0.438620 & 3.6582 & -0.0020 \\
\hline 5 & -0.286568 & -0.526055 & -0.417614 & -0.792154 & 3.5996 & 0.0012 \\
\hline 6 & -0.312783 & -0.525136 & -0.442411 & -0.809875 & 3.5948 & $-6.4 \cdot 10^{-7}$ \\
\hline 7 & -0.394804 & -0.521936 & -0.519495 & -0.809875 & 3.5866 & $1.434 \cdot 10^{-5}$ \\
\hline 8 & -0.735908 & -0.508596 & -0.843279 & -0.809875 & 3.5524 & 0.0011 \\
\hline 9 & -0.735908 & -0.508512 & -0.862556 & -0.809875 & 3.5515 & $8.22 \cdot 10^{-6}$ \\
\hline 10 & -0.735908 & -0.505953 & -0.959070 & -0.809875 & 3.5475 & $2.1953 \cdot 10^{-4}$ \\
\hline 11 & -0.735908 & -0.504368 & -1.026730 & -0.809875 & 3.5446 & $1.2636 \cdot 10^{-4}$ \\
\hline
\end{tabular}

Table 7: Results for the deterministic design optimization

\begin{tabular}{|l|c|}
\hline $\begin{array}{l}\text { Number of SQP Iterations } \\
\text { Total Number of LSF evaluations } \\
\text { (including gradient estimation) }\end{array}$ & 59 \\
\hline
\end{tabular}

Table 8: Performance evaluation for the deterministic design optimization 

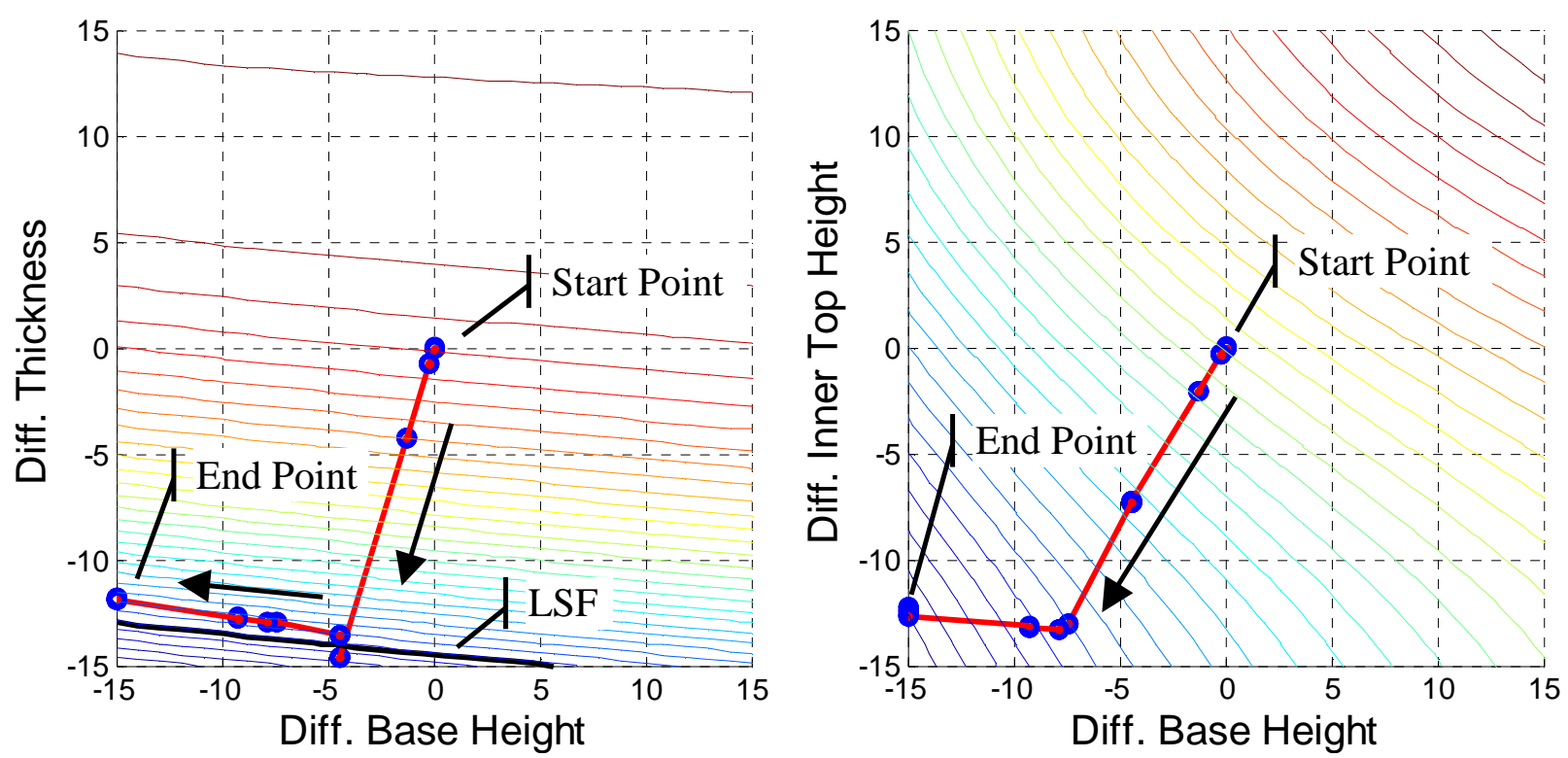

Figure 16: Deterministic Optimization procedure in the standard normal space Y.
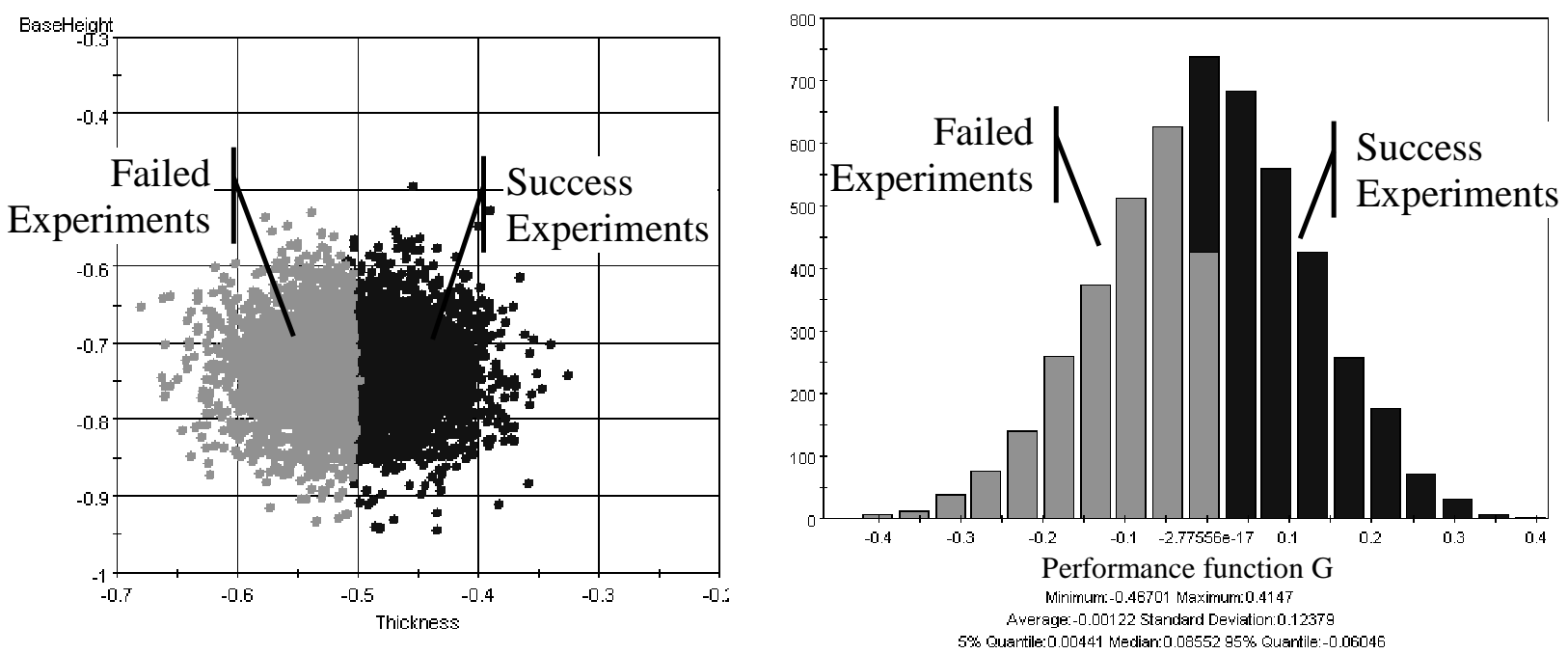

Figure 17: Reliability of the deterministic optimal point.

The second optimization process has been carried out using the Performance Measure Approach and the HMV+ algorithm [28], looking for a robust optimum. The results of the optimization are reported in Table 9 and Table 10 and are shown in Figure 18. 


\begin{tabular}{|c|c|c|c|r|r|r|}
\hline SQP Iteration & $\begin{array}{c}\text { Base Height } \\
(\mathrm{mm})\end{array}$ & $\begin{array}{c}\text { Thickness } \\
(\mathrm{mm})\end{array}$ & $\begin{array}{c}\text { Top Height } \\
(\mathrm{mm})\end{array}$ & $\begin{array}{c}\text { Inner Top Height } \\
(\mathrm{mm})\end{array}$ & $\begin{array}{c}\text { Total Mass } \\
(\mathrm{Kg})\end{array}$ & $\begin{array}{c}\text { Performance } \\
\text { Measure }\end{array}$ \\
\hline Start & -0.735908 & -0.504368 & -1.02673 & -0.809875 & 3.5446 & -0.8071 \\
\hline 1 & -0.735908 & -0.220167 & -1.02584 & -0.809875 & 3.6203 & -0.0319 \\
\hline 2 & -0.735908 & -0.207602 & -1.02673 & -0.809875 & 3.6236 & $-1.39 \cdot 10^{-4}$ \\
\hline
\end{tabular}

Table 9: RBDO results using PMA+/HMV+

\begin{tabular}{|l|l|}
\hline Number of SQP Iterations & 2 \\
\hline Number of LSF evaluations & 280 \\
\hline
\end{tabular}

Table 10: RBDO performance using PMA+/HMV+
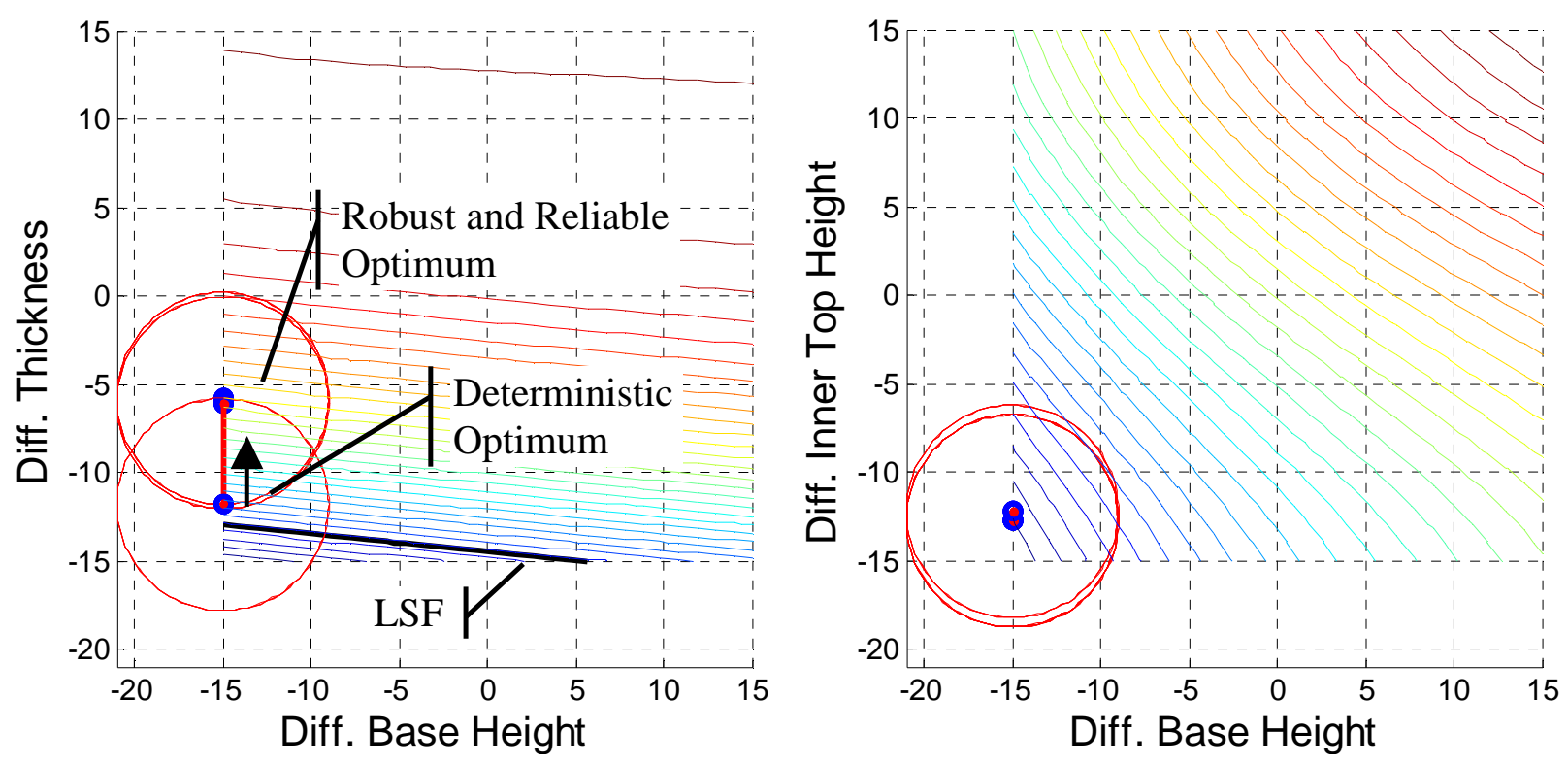

Figure 18: RBDO results in the standard normal space

To demonstrate the efficiency gain for this 2 step optimization strategy, a complete optimization loop starting from the nominal design point and taking into account the probabilistic constraint has been carried out. This optimization procedure required $13 \mathrm{SQP}$ iterations with approximately 985 limit state evaluations, which is 2.9 times more than the actual effort spent with the 2 step approach. For the sake of completeness, this "one step" full process is shown in Figure 19. 

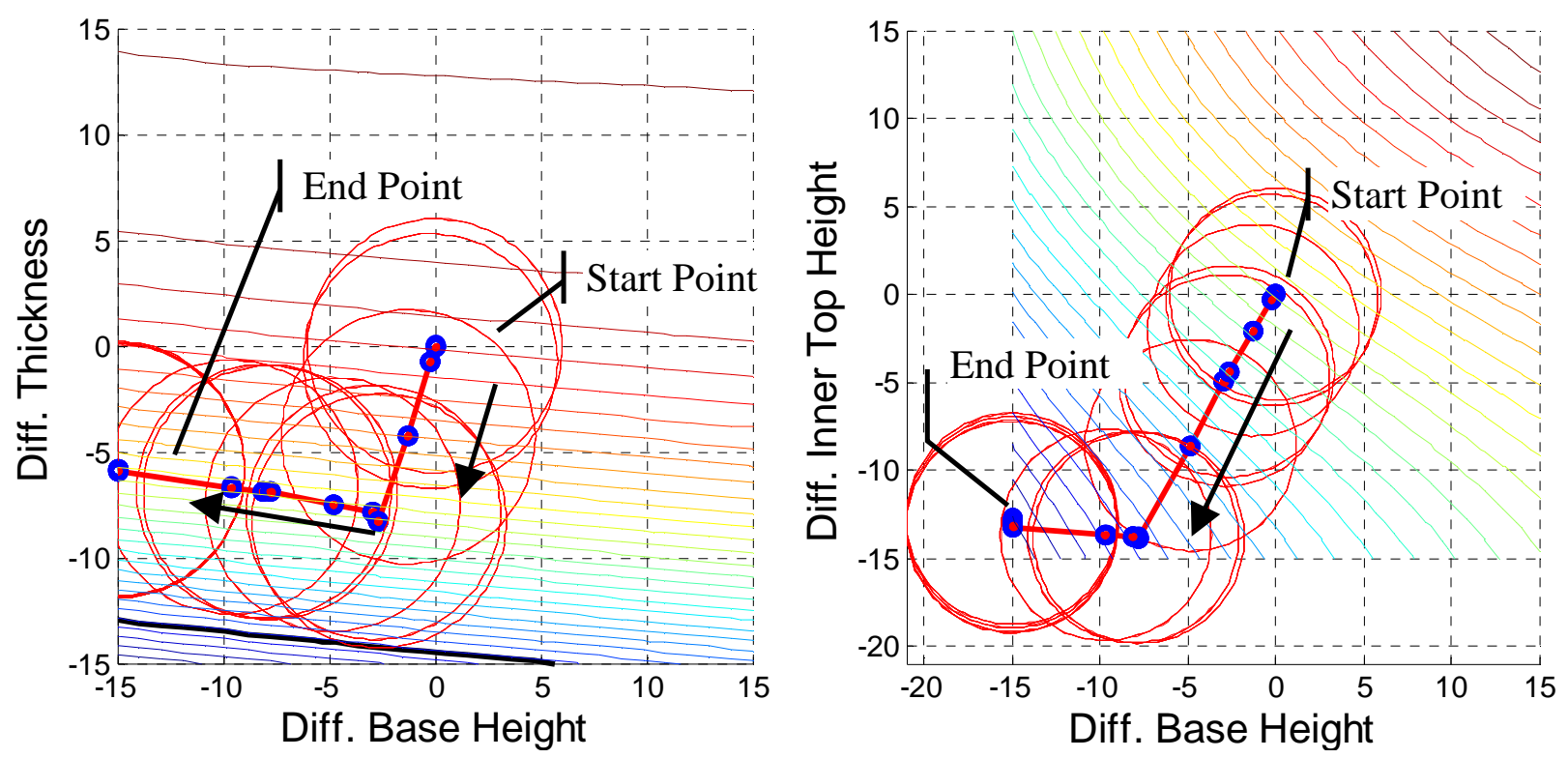

Figure 19: Full RBDO procedure results in the standard normal space

The Reliability-based optimization results clearly show that, in terms of standard deviation, there is room for improvement of the design, while keeping the same reliability. In particular, a negative value in the last row of Table 9 (final optimization point) indicates that the mean values of the parameters reported in Table 1 can be reduced.

The design found with the reliability-based design optimization (which was placed at $6 \sigma$ for the RBDO algorithm using PMA+/HMV+ [26]) shows a mass reduction with respect to the nominal design. This is demonstrated in Table 11 and Table 12.

\begin{tabular}{|c|c|c|c|r|r|r|r|}
\hline & $\begin{array}{c}\text { Base Height } \\
(\mathrm{mm})\end{array}$ & $\begin{array}{c}\text { Thickness } \\
(\mathrm{mm})\end{array}$ & $\begin{array}{c}\text { Top Height } \\
(\mathrm{mm})\end{array}$ & $\begin{array}{c}\text { Inner Top Height } \\
(\mathrm{mm})\end{array}$ & $\begin{array}{c}\text { Total Mass } \\
(\mathrm{kg})\end{array}$ & Safety Factor & $\begin{array}{c}\text { Reliability } \\
\text { Index }\end{array}$ \\
\hline Nominal Point & 0.1518 & 0.1472 & 0.0250 & 0.1027 & 3.9327 & 3.7373 & 14.398 \\
\hline $\begin{array}{c}\text { Deterministic } \\
\text { Optimum }\end{array}$ & -0.7359 & -0.5044 & -1.0267 & -0.8099 & 3.5446 & 2.5001 & 0.0 \\
\hline $\begin{array}{c}\text { Probabilistic } \\
\text { Optimum }\end{array}$ & -0.7359 & -0.2076 & -1.0267 & -0.8099 & 3.6236 & & 6.0 \\
\hline
\end{tabular}

Table 11: Comparison of the results for the 2 optimization procedures.

\begin{tabular}{|c|c|c|}
\hline Nominal Mass & Optimized Mass & Mass reduction \\
\hline 3.9327 & 3.6236 & $7.86 \%$ \\
\hline
\end{tabular}

Table 12: Mass reduction summary. 
This mass reduction has a value of $7.86 \%$ with respect to the nominal mass and constitutes the main result of the optimization. This reduction is particularly significant when one considers that the standard deviations observed in the measured tolerance data are very small.

Even if further reduction of the mass can be achieved by considering larger response exploration, one should pay attention that, when modifications are large in terms of standard deviation, other failure modes may become relevant, which are not important for the current case and therefore can be neglected (e.g. buckling of the left and right thin plates where the Thickness parameter is defined). Thus attention should be given to avoid that the reliability of the structure is improved w.r.t. the considered failure mode and at the same time, other failure modes don't become as relevant as those considered for the reliability analysis.

It is important to note that the time needed for the reliability analysis and the optimization process has been very limited, thanks to the use of the response surface model. In fact each FE computation of the structural and durability analysis takes a total of 40 minutes on a standard PC, while the response surface takes less than a second to evaluate. This makes it very clear that a Monte Carlo simulation and the optimization process would have been much more expensive, in terms of computational effort and time, without the use of a response model.

The integration of the reliability optimization together with the precise manufacturing process used for the slat track thus opens the way for more reliable and light products, while still preserving a very reliable safety margin in the design.

\section{Conclusions}

In this paper, a slat track under variable load conditions has been analyzed considering the variabilities in the input parameters. Several observations have been made.

In a traditional design process, it is not possible to assess the effect of variability on durability performance. As compensation, safety factors are introduced, which unfortunately may lead to slightly over-designed structures. For the slat track, variabilities of the geometric tolerances for 4 parameters have been considered. These tolerances have been parameterized as shape design variables using mesh morphing technology, enabling the accurate assessment of their effect on the durability performance. The entire process (mesh morphing, finite element analysis, durability analysis, reliability assessment and optimization) has been captured in a state-of-the-art process integration and design optimization environment. A design of experiments with response surface methodology has been used to 
speed up the calculation process [29]. A moderately sized design space has been selected, as the paper concerned an assessment and optimization on an already manufactured, extensively tested and validated slat track (and not on a novel design concept for which much larger ranges of variation could be of interest).

A statistical characterization of the selected parameters was done using measured data during the production process, thus enabling the use of reliability analysis techniques. The performance function for the reliability analysis was based on the safety factor prediction of the durability computation. The safety factor has somewhat been lowered when compared to the nominal design, but still quite a high value is used to absolutely guarantee the design safety.

Furthermore, state-of-the-art reliability-based design optimization methods (using PMA+/HMV+ [12]) have been used to take advantage of the margin in the reliability: the design could be re-located in the parameters space at a point where a lighter structure is possible, while still guaranteeing a design reliability of $6 \sigma$. More specifically, for the slat track, a mass reduction of $7.86 \%$ with respect to the nominal mass has been obtained. This is a very significant result of the optimization, especially when one considers that the standard deviations observed in the measured tolerance data are very small. Further gain in the mass reduction can be achieved if a different set-up of the morphing transformation is created and other input parameters are introduced in the design process. Also, attention should be given that other failure modes (e.g. buckling) don't become as relevant as the one considered.

Note that the number of variables and their selection is not limited to the present choice, as they can be changed and increased in number, depending on the particular application. In fact, it is the engineer who should ensure that all relevant tolerance parameters are included in the analysis. Also, a number of techniques are available to handle high dimensional and non-linear problems, where the number of parameters cannot be reduced reasonably without negligible approximations [15]. This methodology and its application are seen as future work.

In summary, the methodology outlined in this paper constitutes an effective general-purpose tool to assess the inherent robustness and reliability of a design. It allows the design robustness and reliability to be improved in accordance with safety regulations, to gain advantages in costs and time-to-market. This methodology can be generalized in the following steps:

- Characterize variability of input parameters with random variables

- Introduce the variability characterization in the engineering process using mesh morphing

- Assess the reliability of the nominal design given the variability of the input parameters 
- Optimize the structure using probabilistic constraints to satisfy safety requirements

These steps may take advantage of a number of analysis tools (DOEs, RSMs etc) [30] that can reduce the computational effort required to satisfy safety requirements.

\section{Acknowledgments}

The presented methodologies are being studied and developed in the context of the EC research training network (RTN) MADUSE (Modelling product vAriability and Data Uncertainty in Structural dynamics Engineering, see http://www.maduse.org/) and the IWT research and development project "Combining test and simulation to improve the reliability of safety critical airplanes components". The support of the European Commission and IWT is gratefully acknowledged.

\section{References}

[1] Oberkampf, W. e.a., Variability, Uncertainty and Error in Computational Simulation, AIAA/ASME Joint Thermophysics and Heat Transfer Conference, ASME-HTD-Vol.357-2, pp. 259-272, 1998.

[2] Oberkampf, W., DeLand, S.M., Rutherford, B.M., Diegert, K.V., Alvin, K.F., Error and uncertainty in modeling and simulation, Reliability Engineering and System Safety 75 (2002) 333-357, Elsevier.

[3] Oberkampf, W., DeLand, S.M., Rutherford, B.M., Diegert, K.V., Alvin, K.F., Estimation of Total Uncertainty in Modeling and Simulation, SANDIA Report SAND2000-0824, Sandia National Laboratories, Albuquerque, New Mexico, USA.

[4] Melchers, R.B., Structural Reliability Analysis and Prediction, $2^{\text {nd }}$ Edition, John Wiley and Sons, 1999.

[5] Der Kiureghian, A., First and Second Order Reliability Methods, Engineering Design Reliability Handbook, CRC Press LLC, 2005.

[6] Thoft-Christensen, P., System Reliability, Engineering Design Reliability Handbook, CRC Press LLC, 2005.

[7] Montgomery, D.C., Runger, G.C., Applied Statistics and Probability for Engineers, John Wiley and Sons, 1994.

[8] Erto, P., Probabilita' e statistica per le scienze e l'ingegneria, $2^{\text {nd }}$ edition, McGraw-Hill, 2003.

[9] Der Kiureghian, A., Liu, P.-L., Optimization Algorithms for Structural Reliability Analysis, Report No. UCB/SESM-86/09, Berkeley University, 1986.

[10] Sudret, B., Der Kiureghian, A., Stochastic Finite Element Methods and Reliability, A state of the art report, Report N. UCB/SEMM-2000/08 Department of Civil and Environmental Engineering, University of California, Berkley, November 2000.

[11] Youn, B.D., Choi, K.K., Park, Y.H., Hybrid Analysis Method for Reliability-Based Design Optimization, Journal of Mechanical Design, vol 125, pp 221-232, June 2003, ASME.

[12] Youn, B.D., Choi, K.K., Du, L., Adaptive Probability Analysis Using An Enhanced Hybrid Mean Value Method, Journal of Structural and Multidisciplinary Optimization, vol. 29, no. 2, 2004, pp. 134-148, Springer Berlin Heidelberg.

[13] d'Ippolito, R., Donders, S., Tzannetakis, N., Van der Auweraer, H., Vandepitte, D., Integration of Probabilistic Methodology in the Aerospace Design Optimization Process, $1^{\text {st }}$ AIAA Multidisciplinary Design Optimization Specialist Conference, Austin, Texas, USA, 18-21 April, 2005 
[14] d'Ippolito, R., Donders, S., Tzannetakis, N., Van de Peer, J., Van der Auweraer, H., An overview of limit state algorithms and their applicability to finite element reliability analysis, $9^{\text {th }}$ International Conference On Structural Safety And Reliability ICOSSAR, Rome, Italy, June 19-23, 2005.

[15] Schuëller, G.I., Pradlwarter, H.J., Koutsourelakis, P.S., A Critical appraisal of reliability estimation procedures for high dimensions, Probabilistic Engineering Mechanics, Vol. 19, pp. 463-474, 2004.

[16] Schlesinger, S., Terminology for Model Credibility, Simulation, Vol. 32, No. 3, 1979.

[17] Computational Fluid Dynamics Committee on Standards, Guide for Verification and Validation of Computational Fluid Dynamics Simulations, American Institute of Aeronautics and Astronautics, AIAA G077-1998, ISBN 1-56347-285-6, January 1998.

[18] Noesis Solutions N.V., OPTIMUS Rev. 5.2, August 2006.

[19] Van der Auweraer, H., Van Langenhove, T., Brughmans, M., El Masri, N., Olbrechts, T., Advanced Mesh Based Design Optimization for Early-Stage Virtual Prototyping, Proc. ISMA 2004, Leuven, Belgium, Sept 20-22, (2004).

[20] Bosmans, I., Brughmans, M., Brizzi, P., Shiozaki, H., Yanase, J., Application of Morphing in Concept Phase of Vehicle Development, Proc. 2005 JSAE Annual Congress, Yokohama, Japan, May 18-20, (2005).

[21] Vecchio, A., Carmine, R., de Voghel, R., Van der Linden, G., Guillaume, P., Numerical Evaluation of Damage Distribution over a Slat Track Using Flight Test Data, Proc. of the $21^{\text {st }}$ International Modal Analysis Conference (IMAC XXI), Orlando, FL, USA 2003.

[22] Barkey, M., Hack, M., Speckert, M., Zingsheim, F., Schäfer, G., LMS.FALANCS Theory Manual, LMS Deutschland GmbH, Luxemburger Straße 7, D-67657 Kaiserslautern, Germany, 2002.

[23] LMS International, LMS Virtual.Lab, Rev. 6B, November 2006.

[24] T.J. Lorenzen, V.L. Anderson, Design of Experiments, a no-name approach, Marcel Dekker Inc., New York, USA, 1993.

[25] Bucher, C., Macke, M., Response Surfaces for Reliability Assessment, Engineering Design Reliability Handbook, CRC Press LLC 2005.

[26] Myers, R.H., Montgomery, D.C., Response Surface Methodology: Process and Product Optimization Using Designed Experiments, Wiley \& Sons, 1995.

[27] Lawson, C.L., Hanson, R.J., Solving Least Squares Problems, SIAM, 1995.

[28] Tu, J., Choi, K.K., A New Study on Reliability Based Design Optimization, ASME Journal of Mechanical Design, Vol. 121, No. 4, 1999, pp. 557-564

[29] d'Ippolito, R.; Hack, M.; Donders, S.; Van der Auweraer, H.; Tzannetakis, N.; Farkas, L.; Desmet, W., Robust and Reliable Fatigue Design of Automotive and Aerospace Structures, to appear, Proc. Fatigue 2007 (6 ${ }^{\text {th }}$ EIS Conference on Durability and Fatigue), Queens' College, Cambridge, UK, March 26-28, 2007

[30] Donders, S.; d'Ippolito, R.; Van der Auweraer, H.; Hack, M.; Tzannetakis, N.; Farkas, L.; Desmet, W., Uncertainty-Based Design in Automotive and Aerospace Engineering, 2007-01-0355, Proc. SAE 2007 World Congress, Detroit, MI, USA, April 16-19, 2007 